Egypt. Acad. J. Biolog. Sci., 13(1):135-153 (2020)

Egyptian Academic Journal of Biological Sciences

A. Entomology

ISSN 1687- 8809

http://eajbsa.journals.ekb.eg/

\title{
Co-Operation Impact Between Gamma Radiation and Spraying Droplets Distribution for Bioinsecticides Controlling Boll Pests on Cotton Plants in Egypt
}

\author{
Reda, A.M. Amer; Rehab, A.A. Dar; M. S. M. Salem and Omnia, Sh. G. Sheba \\ Plant Protection Research Institute, Agric. Res. Center, Dokki, Giza, Egypt.

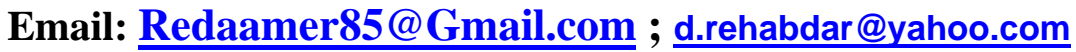

\begin{abstract}
ARTICLE INFO
Article History

Received:19/2/2020

Accepted:10/3/2020

\section{Keywords:}

Gamma radiation, cotton bolls,

Bacillus

thuringiensis,

Diflubenzuron,

Azadirachtin,

Pectinophora

gossypiella,

Earias insulana,

Oxycarenus

hyalinipennis,

Low VOLUME

ground spraying

equipment, cotton

crop.

\section{ABSTRACT}

Field experiments were carried out of about 8 karats planted with (Giza 86) cotton variety during seasons 2018 and 2019 on $21^{\text {st }}$ July and $5^{\text {th }}, 20^{\text {th }}$ August in a field located at Qaha Research Station, Plant Protection Research Institute, Qalyoubia governorate. The selected area was split into 7 plots and the control plot. Two spraying machines were used Pneumatic motor sprayer (Cifarilli) (20 L./Fed.) and Hand-held Hydraulic sprayer (Matabi) (56 L./fed.) for application enhancement. Seven compounds related to different insecticide groups were used; one of them (B. thuringiensis) was exposed to gamma radiation doses of 160, 320 \& 640 Gy (gray) =for potentiating purpose. The treatments were Bacillus thuringiensis (Kurstaki), B. thuringiensis $+160 \mathrm{~Gy}$, B. thuringiensis $+320 \mathrm{~Gy}, B$. thuringiensis +640 Gy, azadirachtin, B. thuringiensis +azadirachtin and diflubenzuron. The treatments mentioned were evaluated against three pests of cotton bolls that were pink bollworm, Pectinophora gossypiella (Saund); spiny bollworm, Earias insulana (Boisd.) and Cottonseed bug, Oxycarenus hyalinipennis (Costa) population and infestation reduction percentages. $B$. thuringiensis +640 Gy and diflubenzuron were considered the best treatments that caused reduction percentages in population and infestations against three pests used, followed by $B$. thuringiensis $+320 \mathrm{~Gy}, B$. thuringiensis +160 Gy and $B$. thuringiensis + azadirachtin, azadirachtin and then $B$. thuringiensis. In addition, the compounds used to enhance the most cotton crop parameters acts in seed numbers, lint and seed weights during the two cotton seasons 2018 \& 2019. So, gamma radiation $(160,320 \& 640 \mathrm{~Gy})$ could potentiate $B$. thuringiensis to become the most effective compound on mentioned three pests and cotton crop parameters compared with the same compounds without exposing to gamma radiation; also, knapsack motor sprayer contribute to success the pest control comparing with Hand-held Hydraulic sprayer (Matabi) (56 L./fed.) that was the least efficient according to homogeneity of droplet spectrum. It could be recommended to use these Bio-Insecticides with Low Volume spraying equipment with not less than (20 L/Fed.). A satisfactory coverage was obtained on cotton plants. The spectrum of droplets ranging between 122-185 $\mu \mathrm{m}$ (VMD). With a sufficient number ranging from $33-253 \mathrm{~N} / \mathrm{cm}^{2}$. The rate of performance of Pneumatic motor sprayer (Cifarilli) (20 L./Fed.) was 12 Fed./day. It was the best equipment, but the lowest rate of performance was Hand-held Hydraulic sprayer (Matabi) (56 L./fed.) since it could spray only 3.45 Fed./day. Data showed that bio-insecticides may be recommended in integrated pest management because of their safety on animals, man and environment. Also, Low Volume spraying reducing the time lost in the process filling the machines with the spray solution and saving the lost spray on the ground.
\end{abstract}




\section{INTRODUCTION}

Cotton (Gossypium barbadense, L.) infested by many economic pests. From these pests were three cotton bolls destructive of pink bollworm, Pectinophora gossypiella (Saund); spiny bollworm, Earias insulana (Boisd.) and Cottonseed bug, Oxycarenus hyalinipennis (Costa) were the most destructive insect pests that cause terrible damage to cotton bolls. P. gossypiella is the destructive pest infesting cotton bolls; the newly hatched penetrates flower buds, flowers, and bolls shortly after hatching and then penetrates the lint and seeds of fully mature bolls (Noble, 1969). While E. insulana is a serious insect pest, it causes a damage to cotton bolls, the larvae mainly feed on fruiting parts of many crops and vegetables causes top boring for the soft and growing tissues especially the terminal buds and later it attack the flower buds and bolls that ultimately shed (Khan, et al. 2007). O. hyalinipennis (adult and nymphs) penetrate the cotton crop (squares and flowers) at an early season, but most economic losses were caused in the late stage (open bolls). Besides damaging the seeds and the reproductive parts, it causes the lint quality resulting in poor ginning of cotton fibers (Ananthakrishan et al., 1982; Sweet, 2000 and Srinivas \& Patil, 2004). Indiscriminate use of synthetic insecticides has not only caused the resistance problem in these pests but it has also polluted the environment. For thus the use of bioagent compounds for controlling the cotton bollworms to try being a successful step in removing chemical pesticides from the environment. Gamma irradiation as a genetic control method was considered among biological methods; it involves the release of genetically modified insects to control the same species (Soon, 1986). Inherited effects of gamma irradiation doses were studied by many authors as Sallam and Ibrahim (1993), Amer (2006), Amer, et al. (2012 and 2018) for controlling P. gossypiella by using B. thuringiensis exposed to different gamma doses that caused potentiating effect against the pest compared to $B$. thuringiensis when used singly without radiation. Rafique et al. (2012) and Abedi, et al (2014) evaluated Azadirachta indica against $P$. gossypilla and $H$. armigera and stated that the compound has insecticidal potential showed significant mortality response.

The first aim of the current field trial is to raise the spraying efficiency by using two spraying machines of Pneumatic motor sprayer (Cifarilli) $(20$ L./Fed.) and Hand-held Hydraulic sprayer (Matabi) (56 L./fed.). Also, the second purpose of this work was to potentiate the compound of B. thuringiensis by exposing to gamma radiation doses of 160 , $320 \& 640$ Gy compared to a bioagent without exposure to gamma doses. In addition, additive compound (B. thuringiensis +azadirachtin) and IGR compound of diflubenzuron were applied. Seven treatments used to control the three insect pests of P. gossypiella, E. insulana and $O$. hyalinipennis on cotton bolls at 2018 and 2019 cotton seasons. A comparative study on the efficiency of different ground sprayers was carried out by (Hindy, 1992 and 1997), who detected a significant variation in the spray deposit due to the arrangement of the nozzles, spray volume, spraying type and rate of application. The world global attention was directed to the minimization of spraying volumes and the control costs which might be happened by using a cheap and effective insecticide or using a developmental ground spraying technique with low application costs per feddan (Magdoline et al. ,1992) and(Mathews,1992). Maintaining sprayers for pesticide application in a good state of repairing and proper working in order to reduce their harmful effects on human health and environment Dokic et al. (2018).

\section{MATERIALS AND METHODS}

Field experiments were carried out of about 8 karats planted with (Giza 86 variety, 2017 strain) cotton variety, planted at $15^{\text {th }}$ Mars during seasons 2018 and 2019, in the field 
located at Qaha Research Station, Plant Protection Research Institute, Qaluobeia governorate. The selected area was split into 7 plots and the control plot. We aimed to evaluate the efficacy of seven treatments to control P. gossypiella, E. insulana, and O. hyalinipennis that infesting cotton bolls during growing cotton seasons 2018 \& 2019. The trial area was divided according to the complete randomized block design including four replicates for each treatment; each treatment was done on one karat each replicate was (1/4 Kirrat). Three rows of cotton plants between treatments left without spraying as barrier zone to avoid drift spray. The experiments were done at $4 \mathrm{pm}$. under local meteorological conditions of $35^{\circ} \mathrm{C}$ average temperature, $60 \%$ average $\mathrm{RH}$ and $2 \mathrm{~m} / \mathrm{sec}$. average wind velocity during the experiment.

The tested compounds were applied three times at 15 days intervals. The first spray was applied when the percent infestation of green bolls reached about 3\% at $21^{\text {th }} \mathrm{July}$, the second spray $5^{\text {th }}$ August and the third spray $20^{\text {th }}$ August at 2018 and 2019 cotton seasons, respectively. Boll samples were collected at random before applying the compounds and then weekly after application. One hundred bolls (25 bolls x 4 replicates) were collected from each treatment and examined.

Insects.

Three pests were investigated on green cotton bolls which mentioned in Table (1).

Table (1). Insects infested the cotton bolls.

\begin{tabular}{|c|c|c|c|}
\hline English name & Bionomial name & Family & Order \\
\hline Pink Bollworm & Pectinophora gossypiella (Saunders) & Gelechiidae & Lepidoptera \\
\hline Spiny Bollworm & Earias insulana (Boisduval) & Noctuidae & Lepidoptera \\
\hline Cotton Seed Bug & Oxycarenus hyalinipennis (Costa) & Lygaeidae & Hemiptera \\
\hline
\end{tabular}

\section{Compounds.}

Seven treatments belong to three compounds as in Table (2).

Table (2). Compounds used common name and application rate.

\begin{tabular}{|c|c|c|c|}
\hline Trade name & Common name & $\begin{array}{l}\text { Application } \\
\text { rate }\end{array}$ & Product Co. \\
\hline $\begin{array}{l}\text { Biotect } 9.4 \% \\
\text { W. P }\end{array}$ & $\begin{array}{l}\text { Bacillus thuringiensis } \\
\text { (Kurstaki) }\end{array}$ & $300 \mathrm{~g} /$ feddan & $\begin{array}{l}\text { Organic for biotechnology co. } \\
\text { Beheira Governorate, Egypt. }\end{array}$ \\
\hline $\begin{array}{c}\text { Achook } \\
0.15 \% \text { EC. }\end{array}$ & $\begin{array}{c}\text { Azadirachtin, } \\
\text { Azadirachta indica }\end{array}$ & $\begin{array}{l}750 \mathrm{~cm}^{3} / \\
\text { feddan }\end{array}$ & Bahar agrochem \& feeds, India \\
\hline $\begin{array}{l}\text { Kllefuron } \\
5 \% \text { EC. }\end{array}$ & Diflubenzuron & $\begin{array}{l}100 \mathrm{~cm}^{3} / \\
\text { feddan }\end{array}$ & $\begin{array}{c}\text { El-Wataneyah Co. For } \\
\text { Agriculture Chemicals } \\
\text { (Agrochem) } \\
\text { Alexandria, Egypt. }\end{array}$ \\
\hline
\end{tabular}

\section{Spraying Equipment Tested on Cotton plants: -} follows:

Two ground application equipment was selected to perform the scope of this work as

1. Hand-held Hydraulic sprayer (Matabi) Spraying volume (56 L./fed.), Espine made.

2. Pneumatic motor sprayer (Cifarilli), Spraying volume (20 L./fed.), Italy made.

- Categorization mentioned in table (3). 
Table 3: Techno-Operational data, calibration and rate of performance of certain ground sprayers applied on Cotton field during season (2018).

\begin{tabular}{|c|c|c|}
\hline Equipment & $\begin{array}{c}\text { Pneumatic motor } \\
\text { Sprayer (Cifarilli) }\end{array}$ & $\begin{array}{c}\text { Hydraulic sprayer } \\
\text { (Matabi) }\end{array}$ \\
\hline Type of atomization & Mechanical Pneumatic & Manual Hydraulic \\
\hline Nozzle type & Air shear nozzle & Hollow cone nozzle $80^{\circ}$ \\
\hline Pump type & Centrifugal fan & Hydraulic pump \\
\hline Number of nozzles & 1 & 5 \\
\hline Pressure (bar) & - & 20 \\
\hline Spray tank (L.) & 20 & 2.4 \\
\hline $\begin{array}{c}\text { Rate of application } \\
\text { (L/fed.) }\end{array}$ & 20 & 1.5 \\
\hline $\begin{array}{c}\text { Working speed } \\
\text { (Km/h.) }\end{array}$ & 2.4 & 0.8 \\
\hline Swath width (m.) & 5 & 0.5 \\
\hline Flow rate (L/min.) & 1 & 0.86 \\
\hline Spray height (m.) & 0.5 & 3.4 \\
\hline Type of Spraying & 2.85 & Target in all sprayers \\
\hline Productivity $*$ (fed./h.) & 12 & \\
\hline $\begin{array}{c}\text { Rate of performance* } \\
\text { (fed./day) }\end{array}$ & \multicolumn{2}{|c|}{} \\
\hline
\end{tabular}

* Number of spraying hours $=8$ hours daily. $*$ Number of workers $=2$.

* Calculations of productivity and rate of performance after Hindy (1992).

\section{Gamma Radiation:}

B. thuringiensis was exposed to gamma radiation doses of 160,320 and $640 \mathrm{~Gy}=$ gray (unit of radiation) at a dose rate of $1.084 \mathrm{KGy} / \mathrm{h}$ by a Cesium ${ }^{137}$ Hendy Gamma Cell Research at National Center for Radiation Researches \& Technology.

\section{Seven Treatments Were Used As Follows:}

1. B. thuringiensis, 2. B. thuringiensis +160 Gy 3. B. thuringiensis +320 Gy, 4. B. thuringiensis $+640 \mathrm{~Gy}$, 5. Azadirachtin, 6. B. thuringiensis +Azadirachtin, 7. Diflubenzuron.

The percent reduction in population and infestation were calculated according to Handerson and Telton formula (1955).

\section{Cotton Crop Parameters:}

The number of seeds and weights of lint and seeds $(\mathrm{g})$ cotton crop were assessed as compared to the control. The samples were collected per 100 open cotton bolls.

\section{Calibration and Performance Adjustment of The Tested Equipment:}

\section{1-Collection of Spray Deposit:}

Before spraying each Cotton treatment, a sampling line constructed of five-wire holder fixed in diagonal line inside each treatment to collect the lost spray between plants; each wire holder top had a fixed water-sensitive paper (Novartis Cards®) on it, also, the water-sensitive paper cards put on five plants; to collect the spray coverage on Cotton leaves at both upper, middle and lower levels of Cotton plant the Cotton plants were about one-meter length, were designed according to Hindy (1989). Cards were collected and transferred carefully inside the paper. Involved data to the laboratory for measuring and calculating the number of droplets $/ \mathrm{cm}^{2}$ and its volume mean diameter (VMD) $\mu \mathrm{m}$ in all treatments was done.

\section{2-Determination of Spray Deposit:}

Number and size of blue spots (deposited droplets) on the water-sensitive papers (Novartis cards $\left.{ }^{\circledR}\right)$ measured with scaled monocular lens (Strüben) ${ }^{\circledR}(15 \mathrm{X})$ Japanese lens. volume mean diameter (VMD) $\mu \mathrm{m}$ and number of droplets in one square centimeter $\left(\mathrm{N} / \mathrm{cm}^{2}\right)$ were estimated according to Hindy (1992). 


\section{Phytotoxic Effect:}

It was determined by recording any color change, leaf curling or flaming up to 15 days after each spraying, according to Badr et al. (1995).

\section{Statistical Analysis:}

All investigated data were analyzed by using Costat statistical program software, 1990 and Duncan multiple range tests (Duncan, 1955) at 5\% probability level to compare the differences among time means.

\section{RESULTS AND DISCUSSION}

A field trial was done at Plant Protection Research Institute Station, Qaha district, Qalubeiah governorate during two cotton seasons (2018 \& 2019). The purpose of the trial was potentiating one compound efficacy (B. thuringiensis) by exposing to gamma radiation doses $(160,320 \& 640 \mathrm{~Gy})$; also, compared its efficacy with the same compounds without radiation; in addition to compare with additive biocides (B. thuringiensis + azedirachtin) and IGR compound (diflubenzuron).

The controlling target pests were pink bollworm, P. gossypiella; spiny bollworm, E. insulana, and cottonseed bug, $O$. hyalinipennis; also, investigate the reduction percentages of larval population and infestation for three pests mentioned. Moreover, determined the cotton crop acts in seed number, lint \& seed weight/100 opened cotton boll during two cotton seasons trials (2018 \& 2019).

Penumatic motor sprayer (Cifarilli) $(20$ L./Fed.) and Hand-held Hydraulic sprayer (Matabi) (56 L./fed.) were used as two spraying machines for obtaining homogenous spray coverage to the compounds applied on cotton plants.

\section{Pink and Spiny Bollworms:}

\section{a. Larval Population Reductions:}

Seven compounds were applied on cotton green bolls when larval population and infestation was $3 \%$ of $P$. gossypiella or E. insulana or both of them.

The pink and spiny larval population reductions had slightly increased at 2019 than 2018 cotton seasons as shown in Tables (3\&4). The IGR compound (diflubenzuron) was the best treatment caused bollworms larval population reduction during two cotton seasons (70.6 $\& 81.1 \%$ and $59.8 \& 72.3 \%$ for Pneumatic motor sprayer (Cifarilli) (20 L./Fed.) and Handheld Hydraulic sprayer (Matabi) (56 L./fed.) during 2018 \& 2019 cotton seasons, respectively). B. thuringiensis $+640 \mathrm{~Gy}$ had population reduction $70.2 \& 79.9 \%$ and $59.1 \&$ $70.2 \%$ for Pneumatic motor sprayer (Cifarilli) (20 L./Fed.) and Hand-held Hydraulic sprayer (Matabi) (56 L./fed.) during 2018 \& 2019 cotton seasons; followed by B. thuringiensis +320 Gy and B. thuringiensis $+160 \mathrm{~Gy}$ as in Tables (4\&5). Moreover, B. thuringiensis + azadirachtin had a potentiating effect in efficacy on larval reduction comparing with each of them when using singly.

\section{b-Infestation Reduction:}

The same trend in larval population reduction was also obvious in bollworms infestation reduction (Tables $6 \& 7$ ); but the treatments used had infestation reduction highly than population reduction.

Also, Pneumatic motor sprayer (Cifarilli) $(20$ L./Fed.) had a high efficacy during compound application comparing with Hand-held Hydraulic sprayer (Matabi) (56 L./fed.). 
Table 4: Percent reduction in larval populations of the pink and spiny bollworms during application by using two spraying machines with some compounds during cotton season 2018 .

\begin{tabular}{|c|c|c|c|c|c|c|c|c|c|c|}
\hline \multirow{3}{*}{ Compounds } & \multicolumn{9}{|c|}{ \% Larval population reductions during application } & \multirow{3}{*}{$\begin{array}{l}\text { Seasonal } \\
\text { Averages }\end{array}$} \\
\hline & \multicolumn{3}{|c|}{$1^{\text {st }}$ spray } & \multicolumn{3}{|c|}{$2^{\text {nd }}$ spray } & \multicolumn{3}{|c|}{$3^{\text {rd }}$ spray } & \\
\hline & 7 & 14 & Aver. & 7 & 14 & Aver. & 7 & 14 & Aver. & \\
\hline \multicolumn{11}{|c|}{ Pneumatic motor sprayer (Cifarilli) $(20 \mathrm{~L} . /$ fed.) } \\
\hline B. thuringiensis & $9.33^{\mathrm{c}}$ & $10.7^{\mathrm{c}}$ & $10.02^{c}$ & $12.6^{\mathrm{c}}$ & $11.1^{\mathrm{c}}$ & $11.9^{\mathrm{c}}$ & $14.3^{\mathrm{d}}$ & $15.2^{\mathrm{c}}$ & $14.8^{\mathrm{c}}$ & $12.2^{\mathrm{c}}$ \\
\hline $\begin{array}{l}\text { B. thuringiensis } \\
+160 \mathrm{~Gy}\end{array}$ & $50^{\mathrm{ab}}$ & $58^{\mathrm{ab}}$ & $5^{b}$ & $58.8^{\mathrm{b}}$ & $66.6^{\mathrm{b}}$ & $62.7^{b}$ & $70^{\mathrm{abc}}$ & $70^{\mathrm{ab}}$ & $70^{\mathrm{ab}}$ & $62.2^{\mathrm{b}}$ \\
\hline $\begin{array}{l}\text { B. thuringiensis } \\
+320 \text { Gy }\end{array}$ & $53.3^{\mathrm{ab}}$ & $60^{\mathrm{ab}}$ & $56.7^{\mathrm{ab}}$ & $62.2^{\mathrm{ab}}$ & $66.6^{b}$ & $64.4^{b}$ & $73^{\mathrm{abc}}$ & $70^{\mathrm{ab}}$ & $71.5^{\mathrm{ab}}$ & $64.2^{\mathrm{ab}}$ \\
\hline $\begin{array}{l}\text { B. thuringiensis } \\
+640 \mathrm{~Gy}\end{array}$ & $58^{\mathrm{a}}$ & $64.4^{\mathrm{a}}$ & $61.2^{\mathrm{a}}$ & $69.9^{\mathrm{a}}$ & $77^{\mathrm{a}}$ & $73.5^{\mathrm{a}}$ & $78^{\mathrm{ab}}$ & $74^{\mathrm{ab}}$ & $76^{\mathrm{a}}$ & $70.2^{\mathrm{a}}$ \\
\hline Azadirachtin & $48.8^{\mathrm{b}}$ & $54.7^{\mathrm{b}}$ & $51.8^{\mathrm{b}}$ & $57^{\mathrm{b}}$ & $59.9^{\mathrm{b}}$ & $58.5^{\mathrm{b}}$ & $67.1^{\mathrm{c}}$ & $62.2^{\mathrm{a}}$ & $64.7^{\mathrm{b}}$ & $58.3^{\mathrm{b}}$ \\
\hline $\begin{array}{l}\text { B. thuringiensis } \\
+ \text { Azadirachtin }\end{array}$ & $48.8^{b}$ & $56.6^{\mathrm{ab}}$ & $52.7^{\mathrm{b}}$ & $58.8^{\mathrm{b}}$ & $63.3^{b}$ & $61.1^{\mathrm{b}}$ & $69^{\mathrm{bc}}$ & $67.1^{\mathrm{b}}$ & $68.1^{\mathrm{ab}}$ & $60.6^{\mathrm{b}}$ \\
\hline Diflubenzuron & $58^{\mathrm{a}}$ & $64.4^{\mathrm{a}}$ & $61.2^{\mathrm{a}}$ & $70^{\mathrm{a}}$ & $77^{\mathrm{a}}$ & $73.5^{\mathrm{a}}$ & $79^{\mathrm{a}}$ & $75^{\mathrm{ab}}$ & $77^{\mathrm{a}}$ & $70.6^{\mathrm{a}}$ \\
\hline L.S.D ${ }_{0.05}$ & 8.21 & 7.31 & 6.32 & 8.95 & 8.74 & 6.19 & 8.74 & 7.54 & 8.65 & 7.54 \\
\hline \multicolumn{11}{|c|}{ Hydraulic Matabi sprayer (56L. IFed.) } \\
\hline B. thuringiensis & $8.88^{\mathrm{c}}$ & $9.9^{\mathrm{d}}$ & $9.39^{\mathrm{c}}$ & $10.8^{\mathrm{c}}$ & $9.5^{c}$ & $10.2^{\mathrm{c}}$ & $11.1^{\mathrm{c}}$ & $12.6^{\mathrm{c}}$ & $11.9^{c}$ & $10.5^{\mathrm{c}}$ \\
\hline $\begin{array}{l}\text { B. thuringiensis } \\
+160 \mathrm{~Gy}\end{array}$ & $40^{\mathrm{b}}$ & $48.8^{\mathrm{bc}}$ & $44.4^{\mathrm{b}}$ & $55^{\mathrm{a}}$ & $61.1^{\mathrm{a}}$ & $58.1^{\mathrm{a}}$ & $60^{\mathrm{ab}}$ & $60^{\mathrm{a}}$ & $60^{\mathrm{a}}$ & $54.2^{\mathrm{ab}}$ \\
\hline $\begin{array}{l}\text { B. thuringiensis } \\
+320 \mathrm{~Gy}\end{array}$ & $44.4^{\mathrm{ab}}$ & $50^{\mathrm{bc}}$ & $47.2^{\mathrm{ab}}$ & $57^{\mathrm{a}}$ & $60^{\mathrm{a}}$ & $61.8^{\mathrm{a}}$ & $60^{\mathrm{ab}}$ & $58^{\mathrm{a}}$ & $59^{\text {a }}$ & $56^{\mathrm{a}}$ \\
\hline $\begin{array}{l}\text { B. thuringiensis } \\
+640 \mathrm{~Gy}\end{array}$ & $48.8^{\mathrm{a}}$ & $55^{\mathrm{ab}}$ & $51.9^{\mathrm{a}}$ & $60^{\mathrm{a}}$ & $66.6^{\mathrm{a}}$ & $63.3^{\mathrm{a}}$ & $64.4^{\mathrm{a}}$ & $60^{\mathrm{a}}$ & $62.2^{\mathrm{a}}$ & $59.1^{\mathrm{a}}$ \\
\hline Azadirachtin & $38^{\mathrm{b}}$ & $44.4^{c}$ & $41.2^{\mathrm{b}}$ & $45.5^{\mathrm{b}}$ & $47^{b}$ & $46.3^{b}$ & $55^{b}$ & $50^{b}$ & $\mathbf{5 2 . 5}^{\mathrm{b}}$ & $46.7^{b}$ \\
\hline $\begin{array}{l}\text { B. thuringiensis } \\
+ \text { Azadirachtin }\end{array}$ & $38^{b}$ & $45^{\mathrm{c}}$ & $41.5^{b}$ & $55^{\mathrm{a}}$ & $59^{\mathrm{a}}$ & $57^{\mathrm{a}}$ & $61.1^{\mathrm{ab}}$ & $62.2^{\mathrm{a}}$ & $61.7^{\mathrm{a}}$ & $53.4^{\mathrm{ab}}$ \\
\hline Diflubenzuron & $48.8^{\mathrm{a}}$ & $59^{\mathrm{a}}$ & $53.9^{\mathrm{a}}$ & $60^{\mathrm{a}}$ & $66.6^{\mathrm{a}}$ & $63.3^{\mathrm{a}}$ & $64.4^{\mathrm{a}}$ & $60^{\mathrm{a}}$ & $62.2^{\mathrm{a}}$ & $59.8^{\mathrm{a}}$ \\
\hline L.S.D ${ }_{0.05}$ & 8.08 & 6.11 & 6.402 & 6.14 & 8.62 & 8.74 & 6.402 & 7.54 & 6.37 & 8.26 \\
\hline
\end{tabular}

Table 5: Percent reduction in larval populations of the pink and spiny bollworms during application by using two spraying machines with some compounds during the cotton season 2019.

\begin{tabular}{|c|c|c|c|c|c|c|c|c|c|c|}
\hline \multirow{3}{*}{ Compounds } & \multicolumn{9}{|c|}{ \% Larval population reductions during application } & \multirow{3}{*}{$\begin{array}{l}\text { Seasonal } \\
\text { Averages }\end{array}$} \\
\hline & \multicolumn{3}{|c|}{$1^{\text {st }}$ spray } & \multicolumn{3}{|c|}{$2^{\text {nd }}$ spray } & \multicolumn{3}{|c|}{$3^{\text {rd }}$ spray } & \\
\hline & 7 & 14 & Aver. & 7 & 14 & Aver. & 7 & 14 & Aver. & \\
\hline \multicolumn{11}{|c|}{ Pneumatic motor sprayer sprayer (Cifarilli) $(20$ L./fed.) } \\
\hline B. thuringiensis & $12.6^{\mathrm{d}}$ & $12^{\mathrm{d}}$ & $12.3^{\mathrm{d}}$ & $14.2^{\mathrm{d}}$ & $16.9^{\mathrm{d}}$ & $15.6^{\mathrm{e}}$ & $20.7^{\mathrm{d}}$ & $20.7^{\mathrm{d}}$ & $20.7^{d}$ & $16.2^{\mathrm{d}}$ \\
\hline $\begin{array}{l}\text { B. thuringiensis } \\
+160 \mathrm{~Gy}\end{array}$ & $60^{\mathrm{abc}}$ & $68.8^{\mathrm{ab}}$ & $64.4^{\mathrm{ab}}$ & $75.5^{\mathrm{ab}}$ & $78^{b}$ & $76.8^{\mathrm{bc}}$ & $81^{\mathrm{abc}}$ & $80^{b}$ & $80.5^{b}$ & $73.9^{\mathrm{ab}}$ \\
\hline $\begin{array}{l}\text { B. thuringiensis } \\
+320 \mathrm{~Gy}\end{array}$ & $64.4^{\mathrm{ab}}$ & $68.8^{\mathrm{ab}}$ & $66.6^{\mathrm{ab}}$ & $79.9^{\mathrm{a}}$ & $81^{\mathrm{ab}}$ & $80.5^{\mathrm{ab}}$ & $86^{\mathrm{ab}}$ & $80^{b}$ & $83^{\mathrm{ab}}$ & $76.7^{\mathrm{ab}}$ \\
\hline $\begin{array}{l}\text { B. thuringiensis } \\
+640 \mathrm{~Gy}\end{array}$ & $66.6^{a}$ & $72^{a}$ & $69.3^{\mathrm{a}}$ & $83^{\mathrm{a}}$ & $85^{a}$ & $84^{\mathrm{a}}$ & $89^{a}$ & $84^{a b}$ & $86.5^{\mathrm{ab}}$ & $79.9^{a}$ \\
\hline Azadirachtin & $52.2^{c}$ & $54.4^{c}$ & $53.3^{c}$ & $62.2^{c}$ & $68.9^{c}$ & $65.6^{d}$ & $72.2^{c}$ & $68.9^{c}$ & $70.6^{c}$ & $63.2^{c}$ \\
\hline $\begin{array}{l}\text { B. thuringiensis } \\
\text { +Azadirachtin }\end{array}$ & $56.6^{b c}$ & $60.6^{b c}$ & $58.6^{\mathrm{bc}}$ & $70.7^{b}$ & $75.5^{b}$ & $73.1^{c}$ & $79^{b c}$ & $78.2^{b}$ & $78.6^{b}$ & $70.1^{b c}$ \\
\hline Diflubenzuron & $66.6^{a}$ & $74^{a}$ & $70.3^{a}$ & $83^{a}$ & $86^{a}$ & $84.5^{\mathrm{a}}$ & $90^{\mathrm{a}}$ & $87^{a}$ & $88.5^{\mathrm{a}}$ & $81.1^{\mathrm{a}}$ \\
\hline L.S.D 0.05 & 7.54 & 8.68 & 8.43 & 7.39 & 6.402 & 6.11 & 8.62 & 6.11 & 7.39 & 8.26 \\
\hline \multicolumn{11}{|c|}{ Hydraulic Matabi sprayer (56L.|Fed.) } \\
\hline B. thuringiensis & $12^{\mathrm{c}}$ & $11^{\mathrm{d}}$ & $11.5^{c}$ & $13^{\mathrm{c}}$ & $15^{e}$ & $14^{c}$ & $17^{\mathrm{d}}$ & $15^{\mathrm{e}}$ & $16^{\mathrm{d}}$ & $13.8^{\mathrm{d}}$ \\
\hline $\begin{array}{l}\text { B. thuringiensis } \\
+160 \mathrm{~Gy}\end{array}$ & $54^{\mathrm{ab}}$ & $59.9^{\mathrm{abc}}$ & $56.95^{\mathrm{ab}}$ & $68.8^{\mathrm{a}}$ & $70^{\mathrm{bcd}}$ & $69.4^{\mathrm{ab}}$ & $75^{b}$ & $70^{\mathrm{b}}$ & $72.5^{b}$ & $66.3^{\mathrm{abc}}$ \\
\hline $\begin{array}{l}\text { B. thuringiensis } \\
+320 \mathrm{~Gy}\end{array}$ & $56^{\mathrm{ab}}$ & $59.9^{\mathrm{abc}}$ & $57.95^{\mathrm{ab}}$ & $70^{\mathrm{a}}$ & $74^{\mathrm{abc}}$ & $72^{\mathrm{a}}$ & $80^{\mathrm{a}}$ & $70^{b}$ & $75^{\mathrm{ab}}$ & $68.3^{\mathrm{ab}}$ \\
\hline $\begin{array}{l}\text { B. thuringiensis } \\
+640 \mathrm{~Gy}\end{array}$ & $60^{\mathrm{a}}$ & $62.2^{\mathrm{ab}}$ & $61.1^{\mathrm{a}}$ & $72^{\mathrm{a}}$ & $77^{\mathrm{ab}}$ & $74.5^{\mathrm{a}}$ & $80^{\mathrm{a}}$ & $70^{\mathrm{b}}$ & $75^{\mathrm{ab}}$ & $70.2^{\mathrm{ab}}$ \\
\hline Azadirachtin & $50^{b}$ & $52.2^{c}$ & $51.1^{\mathrm{b}}$ & $59^{b}$ & $64.4^{\mathrm{d}}$ & $61.7^{b}$ & $68.8^{c}$ & $59^{d}$ & $63.9^{c}$ & $58.9^{c}$ \\
\hline $\begin{array}{l}\text { B. thuringiensis } \\
\text { +Azadirachtin }\end{array}$ & $53^{b}$ & $55^{\mathrm{bc}}$ & $54^{\mathrm{ab}}$ & $65^{\mathrm{ab}}$ & $69^{\mathrm{cd}}$ & $67^{\mathrm{ab}}$ & $74^{b}$ & $65^{c}$ & $69.5^{\text {bc }}$ & $63.5^{b c}$ \\
\hline Diflubenzuron & $60^{a}$ & $65^{a}$ & $62.5^{a}$ & $72^{\mathrm{a}}$ & $78^{a}$ & $75^{a}$ & $82^{a}$ & $77^{\mathrm{a}}$ & $79.5^{\mathrm{a}}$ & $72.3^{a}$ \\
\hline L.S.D 0.05 & 6.15 & 7.89 & 8.003 & 7.59 & 7.29 & 7.53 & 4.03 & 4.34 & 6.24 & 7.47 \\
\hline
\end{tabular}

Numbers followed by the same letter at the same column are not significantly different at $P=0.05$. 
Table 6: Infestation percent reductions of the pink and spiny bollworms during application by using two spraying machines with some compounds during cotton season 2018 .

\begin{tabular}{|c|c|c|c|c|c|c|c|c|c|c|}
\hline \multirow{3}{*}{ Compounds } & \multicolumn{9}{|c|}{ \% Infestation reductions during application } & \multirow{3}{*}{$\begin{array}{l}\text { Seasonal } \\
\text { Averages }\end{array}$} \\
\hline & \multicolumn{3}{|c|}{$1^{\text {st }}$ spray } & \multicolumn{3}{|c|}{$2^{\text {nd }}$ spray } & \multicolumn{3}{|c|}{$3^{\text {rd }}$ spray } & \\
\hline & 7 & 14 & Aver. & 7 & 14 & Aver. & 7 & 14 & Aver. & \\
\hline \multicolumn{11}{|c|}{ Pneumatic motor spraver (Cifarilli) (20 L./fed.) } \\
\hline B. thuringiensis & $7.32^{\mathrm{d}}$ & $8.08^{\mathrm{e}}$ & $7.7^{\mathrm{e}}$ & $13.6^{\mathrm{d}}$ & $17.1^{\mathrm{d}}$ & $15.4^{\mathrm{d}}$ & $20.2^{\mathrm{d}}$ & $20.2^{\mathrm{d}}$ & $20.2^{\mathrm{d}}$ & $14.4^{\mathrm{d}}$ \\
\hline $\begin{array}{l}\text { B. thuringiensis } \\
+160 \mathrm{~Gy}\end{array}$ & $60^{\mathrm{b}}$ & $66.6^{\mathrm{bc}}$ & $63.3^{\text {bc }}$ & $67^{\mathrm{b}}$ & $69.9^{\mathrm{ab}}$ & $68.5^{\mathrm{ab}}$ & $72.2^{\mathrm{b}}$ & $66.6^{\mathrm{bc}}$ & $69.4^{b}$ & $67.1^{\mathrm{b}}$ \\
\hline $\begin{array}{l}\text { B. thuringiensis } \\
+320 \mathrm{~Gy}\end{array}$ & $66.6^{\mathrm{ab}}$ & $69.9^{\mathrm{ab}}$ & $68.3^{\mathrm{ab}}$ & $72.2^{\mathrm{ab}}$ & $72.2^{\mathrm{ab}}$ & $72.2^{\mathrm{ab}}$ & $76^{\mathrm{ab}}$ & $70^{\mathrm{b}}$ & $73^{\mathrm{ab}}$ & $71.2^{\mathrm{ab}}$ \\
\hline $\begin{array}{l}\text { B. thuringiensis } \\
+640 \mathrm{~Gy}\end{array}$ & $72.2^{\mathrm{a}}$ & $72.2^{\mathrm{ab}}$ & $72.2^{2}$ & $76^{2}$ & $77^{\mathrm{a}}$ & $76.5^{\mathrm{a}}$ & $82^{\mathrm{a}}$ & $80^{\mathrm{a}}$ & $81^{\mathrm{a}}$ & $76.6^{\mathrm{a}}$ \\
\hline Azadirachtin & $50^{\mathrm{c}}$ & $52.2^{\mathrm{d}}$ & 51.1 ${ }^{\mathrm{d}}$ & $54^{\mathrm{c}}$ & $56^{\mathrm{c}}$ & $55^{\mathrm{c}}$ & $60^{\mathrm{c}}$ & $58.8^{\mathrm{c}}$ & $59.4^{\mathrm{c}}$ & $55.2^{\mathrm{c}}$ \\
\hline $\begin{array}{l}\text { B. thuringiensis } \\
\text { +Azadirachtin }\end{array}$ & $60^{b}$ & $58.8^{\mathrm{cd}}$ & $59.4^{\mathrm{c}}$ & $64.4^{b}$ & $66.6^{\mathrm{b}}$ & $65.5^{b}$ & $69.9^{\mathrm{b}}$ & $58.8^{c}$ & $64.4^{\mathrm{bc}}$ & $63.1^{\mathrm{bc}}$ \\
\hline Diflubenzuron & $69.6^{a}$ & $78^{\mathrm{a}}$ & $73.8^{\mathrm{a}}$ & $77^{\mathrm{a}}$ & $78^{\mathrm{a}}$ & $77.5^{\mathrm{a}}$ & $84^{\mathrm{a}}$ & $80^{\mathrm{a}}$ & $82^{\mathrm{a}}$ & $77.8^{\mathrm{a}}$ \\
\hline L.S.D $\mathbf{D}_{0.05}$ & 7.54 & 8.68 & 7.87 & 8.43 & 8.95 & 8.39 & 7.72 & 8.003 & 8.74 & 8.62 \\
\hline \multicolumn{11}{|c|}{ Hydraulic Matabi sprayer (56L.|Fed.) } \\
\hline B. thuringiensis & $7^{\mathrm{d}}$ & $7.2^{\mathrm{d}}$ & $7.1^{c}$ & $11.1^{\mathrm{e}}$ & $15.5^{\mathrm{d}}$ & $13.3^{\mathrm{e}}$ & $17.7^{\mathrm{c}}$ & $17^{\mathrm{d}}$ & $17.4^{\mathrm{d}}$ & $12.6^{\mathrm{e}}$ \\
\hline $\begin{array}{l}\text { B. thuringiensis } \\
+160 \mathrm{~Gy}\end{array}$ & $50^{\mathrm{bc}}$ & $55^{\mathrm{abc}}$ & $52.5^{\mathrm{ab}}$ & $56^{\mathrm{cd}}$ & $58.8^{\mathrm{b}}$ & $57.4^{\mathrm{cd}}$ & $60^{\mathrm{b}}$ & $55^{\mathrm{bc}}$ & $57.5^{\mathrm{c}}$ & $55.8^{\mathrm{cd}}$ \\
\hline $\begin{array}{l}\text { B. thuringiensis } \\
+320 \mathrm{~Gy}\end{array}$ & $53^{\mathrm{ab}}$ & $55^{\mathrm{abc}}$ & $54^{\mathrm{ab}}$ & $60^{\mathrm{bc}}$ & $62.2^{\mathrm{b}}$ & $61.1^{\mathrm{bc}}$ & $70^{\mathrm{a}}$ & $60^{\mathrm{b}}$ & $65^{\mathrm{b}}$ & $60.03^{\text {bc }}$ \\
\hline $\begin{array}{l}\text { B. thuringiensis } \\
+640 \mathrm{~Gy}\end{array}$ & $55^{\mathrm{ab}}$ & $58.8^{\mathrm{ab}}$ & $56.9^{\mathrm{ab}}$ & $66.6^{\mathrm{ab}}$ & $70^{\mathrm{a}}$ & $68.3^{\mathrm{ab}}$ & $72.2^{\mathrm{a}}$ & $70^{\mathrm{a}}$ & $71.1^{\mathrm{ab}}$ & $65.4^{\mathrm{ab}}$ \\
\hline Azadirachtin & $46^{\mathrm{c}}$ & $50^{\mathrm{c}}$ & $48^{\mathrm{b}}$ & $50^{\mathrm{d}}$ & $48^{\mathrm{c}}$ & $49^{d}$ & $53.3^{\mathrm{b}}$ & $50^{\mathrm{c}}$ & $51.7^{\mathrm{c}}$ & $49.6^{d}$ \\
\hline $\begin{array}{l}\text { B. thuringiensis } \\
\text { +Azadirachtin }\end{array}$ & $50^{\mathrm{bc}}$ & $52^{\mathrm{bc}}$ & $51^{b}$ & $52^{\mathrm{cd}}$ & $55^{\mathrm{bc}}$ & $53.5^{\mathrm{cd}}$ & $56.6^{b}$ & $50^{c}$ & $53.3^{c}$ & $52.6^{\text {cd }}$ \\
\hline Diflubenzuron & $58.8^{\mathrm{a}}$ & $62.2^{\mathrm{a}}$ & $60.5^{3}$ & $70^{\mathrm{a}}$ & $73^{a}$ & $71.5^{\mathrm{a}}$ & $75^{\mathrm{a}}$ & $70^{\mathrm{a}}$ & $72.5^{\mathrm{a}}$ & $68.2^{2}$ \\
\hline L.S.D 0.05 & 6.402 & 7.26 & 8.43 & 7.74 & 7.72 & 8.88 & 7.59 & 6.14 & 6.47 & 7.26 \\
\hline
\end{tabular}

Numbers followed by the same letter at the same column are not significantly different at $P=0.05$.

Table 7: Infestation percent reductions of the pink and spiny bollworms during application by using two spraying machines with some compounds during the cotton season 2019.

\begin{tabular}{|c|c|c|c|c|c|c|c|c|c|c|}
\hline \multirow{3}{*}{ Compounds } & \multicolumn{9}{|c|}{ \% Infestation reductions during application } & \multirow{3}{*}{$\begin{array}{l}\text { Seasona } \\
\text { Average }\end{array}$} \\
\hline & \multicolumn{3}{|c|}{$1^{\text {st }}$ spray } & \multicolumn{3}{|c|}{$2^{\text {nd }}$ spray } & \multicolumn{3}{|c|}{$3^{\text {rd }}$ spray } & \\
\hline & 7 & 14 & Aver. & 7 & 14 & Aver. & 7 & 14 & Aver. & \\
\hline \multicolumn{11}{|c|}{ Pneumatic motor sprayer (Cifarilli) (20 L./fed.) } \\
\hline B. thuringiensis & $10^{\mathrm{d}}$ & $12^{\mathrm{e}}$ & $11^{\mathrm{e}}$ & $20.2^{\mathrm{f}}$ & $23.9^{\mathrm{e}}$ & $22.1^{\mathrm{e}}$ & $33.3^{\mathrm{d}}$ & $24.4^{\mathrm{d}}$ & $28.9^{\mathrm{d}}$ & $20.7^{\mathrm{d}}$ \\
\hline $\begin{array}{l}\text { B. thuringiensis } \\
+160 \mathrm{~Gy}\end{array}$ & $60^{\mathrm{b}}$ & $70^{\mathrm{bc}}$ & $\mathbf{6 5}^{\mathrm{bc}}$ & $70^{\text {cd }}$ & $82.2^{\mathrm{bc}}$ & $76.1^{\mathrm{c}}$ & $86^{\mathrm{ab}}$ & $80^{\mathrm{b}}$ & $\mathbf{8 3}^{\mathrm{b}}$ & $74.7^{b}$ \\
\hline $\begin{array}{l}\text { B. thuringiensis } \\
+320 \mathrm{~Gy}\end{array}$ & $66.6^{\mathrm{ab}}$ & $75^{\mathrm{ab}}$ & $70.8^{\mathrm{ab}}$ & $78^{\mathrm{bc}}$ & $85^{\mathrm{abc}}$ & $81.5^{\text {bc }}$ & $89^{a b}$ & $83^{\mathrm{ab}}$ & $86^{\mathrm{ab}}$ & $79.4^{\mathrm{ab}}$ \\
\hline $\begin{array}{l}\text { B. thuringiensis } \\
+640 \mathrm{~Gy}\end{array}$ & $70^{\mathrm{a}}$ & $80^{\mathrm{a}}$ & $75^{\mathrm{a}}$ & $84^{\mathrm{ab}}$ & $90^{a b}$ & $87^{a b}$ & $90^{a b}$ & $85^{\mathrm{ab}}$ & $87.5^{\mathrm{ab}}$ & $83.2^{\mathrm{a}}$ \\
\hline Azadirachtin & $52.2^{\mathrm{c}}$ & $56^{d}$ & $54.1^{\mathrm{d}}$ & $60^{\mathrm{e}}$ & $60^{d}$ & $60^{d}$ & $64^{c}$ & $62.8^{\mathrm{c}}$ & $63.4^{\mathrm{c}}$ & $59.2^{\mathrm{c}}$ \\
\hline $\begin{array}{l}\text { B. thuringiensis } \\
\text { +Azadirachtin }\end{array}$ & $60^{\mathrm{b}}$ & $65.5^{\mathrm{c}}$ & $62.8^{\mathrm{c}}$ & $69.5^{\mathrm{d}}$ & $78^{c}$ & $73.8^{\mathrm{c}}$ & $82.2^{\mathrm{b}}$ & $78.8^{b}$ & $80.5^{b}$ & $72.4^{b}$ \\
\hline Diflubenzuron & $70^{\mathrm{a}}$ & $80^{\mathrm{a}}$ & $7^{\mathrm{a}}$ & $88^{\mathrm{a}}$ & $92^{\mathrm{a}}$ & $90^{\mathrm{a}}$ & $93^{a}$ & $90^{\mathrm{a}}$ & $91.5^{\mathrm{a}}$ & $85.5^{\mathrm{a}}$ \\
\hline L.S.D 0.05 & 6.32 & 7.39 & 6.22 & 8.08 & 8.39 & 7.78 & 7.54 & 7.59 & 7.47 & 88 \\
\hline \multicolumn{11}{|c|}{ Hydraulic Matabi sprayer (56L.IFed.) } \\
\hline B. thuringiensis & $9^{\mathrm{c}}$ & $10^{\mathrm{d}}$ & $9.5^{\mathrm{c}}$ & $16^{\mathrm{e}}$ & $17^{\mathrm{e}}$ & $16.5^{\mathrm{e}}$ & $28^{\mathrm{e}}$ & $18^{\mathrm{d}}$ & $23^{\mathrm{d}}$ & $16.3^{\mathrm{d}}$ \\
\hline $\begin{array}{l}\text { B. thuringiensis } \\
+160 \mathrm{~Gy}\end{array}$ & $55^{\mathrm{ab}}$ & $60^{\mathrm{b}}$ & $\mathbf{5 7 . 5}^{\mathrm{ab}}$ & $62.2^{\mathrm{c}}$ & $66.6^{\mathrm{c}}$ & $64.4^{c}$ & $75^{\text {bc }}$ & $70^{\mathrm{b}}$ & $72.5^{\mathrm{b}}$ & $64.8^{b}$ \\
\hline $\begin{array}{l}\text { B. thuringiensis } \\
+320 \mathrm{~Gy}\end{array}$ & $58.8^{\mathrm{a}}$ & $65^{\mathrm{ab}}$ & $61.9^{\mathrm{a}}$ & $66.6^{\mathrm{bc}}$ & $70^{\mathrm{bc}}$ & $68.3^{\text {bc }}$ & $80^{a b}$ & $75^{\mathrm{ab}}$ & $77.5^{\mathrm{ab}}$ & $69.2 \mathrm{ab}$ \\
\hline $\begin{array}{l}\text { B. thuringiensis } \\
+640 \mathrm{~Gy}\end{array}$ & $60^{\mathrm{a}}$ & $70^{\mathrm{a}}$ & $65^{\mathrm{a}}$ & $72^{\mathrm{ab}}$ & $75^{\mathrm{ab}}$ & 73.5 $5^{\mathrm{ab}}$ & $82^{a b}$ & $80^{\mathrm{a}}$ & $81^{\mathrm{a}}$ & $73.2^{\mathrm{a}}$ \\
\hline Azadirachtin & $50^{\mathrm{b}}$ & $50^{\mathrm{c}}$ & $5^{b}{ }^{b}$ & $50^{\mathrm{d}}$ & $55^{\mathrm{d}}$ & $\mathbf{5 2 . 5 ^ { d }}$ & $60^{d}$ & $59^{\mathrm{c}}$ & $59.5^{\mathrm{c}}$ & $54^{\mathrm{c}}$ \\
\hline $\begin{array}{l}\text { B. thuringiensis } \\
\text { +Azadirachtin }\end{array}$ & $55^{\mathrm{ab}}$ & $58.8^{\mathrm{b}}$ & $56.9^{\mathrm{ab}}$ & $60^{c}$ & $64^{c}$ & $62^{c}$ & $70^{c}$ & $70^{b}$ & $70^{b}$ & $62.9^{b}$ \\
\hline Diflubenzuron & $60^{\mathrm{a}}$ & $70^{\mathrm{a}}$ & $65^{\mathrm{a}}$ & $75^{\mathrm{a}}$ & $78^{\mathrm{a}}$ & $76.5^{a}$ & $84^{\mathrm{a}}$ & $80^{\mathrm{a}}$ & $82^{\mathrm{a}}$ & $74.5^{\mathrm{a}}$ \\
\hline L.S.D 0.05 & 6.14 & 6.66 & 7.74 & 7.98 & 6.11 & 6.08 & 7.54 & 8.003 & 7.89 & 6.05 \\
\hline
\end{tabular}

Numbers followed by the same letter at the same column are not significantly different at $P=0.05$. 


\section{Cotton Seed Bug:}

\section{a. Population:}

Tables (8\&9) showed that diflubenzuron as well as B. thuringiensis +640 Gy nearly was considered the best treatments caused reduction in seed bug population, followed by $B$. thuringiensis $+320 \mathrm{~Gy}$ and $B$. thuringiensis $+160 \mathrm{~Gy}$ had potentiating efficacy on cottonseed bug population than its reduction on cottonseed bug without exposing to gamma doses. Moreover, B. thuringiensis + azadirachtin had the best efficacy compared to use each of them singly.

\section{b. Infestation.}

The previous trend has also appeared in cottonseed bug infestation reduction as described in Tables (10\&11).

Shah, et al. (2016) assess the efficacy of 12 insecticides against dusky cotton bug (Oxycarenus laetus) in field conditions, the insecticides were belonging to five different groups viz. organophosphate, pyrethroid, neo-nicotinoid, naturalize and insect growth regulator (IGRs). All tested insecticides were significantly different in relation to pest mortality than the untreated check. Among the insecticides, organophosphates proved the most effective, followed by pyrethroids and neonicotinoids while naturalize proved least effective, followed by insect growth regulator (IGRs).

Table 8: Percent reduction in cottonseed bug populations during application by using two spraying machines with some compounds during the cotton season 2018

\begin{tabular}{|c|c|c|c|c|c|c|c|c|c|c|}
\hline \multirow{3}{*}{ Compounds } & \multicolumn{9}{|c|}{$\%$ Reduction of cotton seed bug populations during application } & \multirow{3}{*}{$\begin{array}{l}\text { Seasonal } \\
\text { Average }\end{array}$} \\
\hline & \multicolumn{3}{|c|}{$1^{\text {st }}$ spray } & \multicolumn{3}{|c|}{$2^{\text {nd }}$ spray } & \multicolumn{3}{|c|}{$3^{\text {rd }}$ sprav } & \\
\hline & 7 & 14 & Aver. & 7 & 14 & Aver. & 7 & 14 & Aver. & \\
\hline \multicolumn{11}{|c|}{ Pneumatic motor sprayer (Cifarilli) (20 L./fed.) } \\
\hline B. thuringiensis & $20^{c}$ & $25^{\mathrm{c}}$ & $22.5^{\mathrm{d}}$ & $25^{\mathrm{d}}$ & $35^{\mathrm{d}}$ & $30^{\mathrm{d}}$ & $11.8^{\mathrm{d}}$ & $10^{d}$ & $10.9^{\mathrm{d}}$ & $21.1^{\mathrm{d}}$ \\
\hline $\begin{array}{l}\text { B. thuringiensis } \\
+160 \mathrm{~Gy}\end{array}$ & $38^{\mathrm{a}}$ & $46.6^{b}$ & $42.3^{\mathrm{abc}}$ & $58.8^{c}$ & $69.9^{\mathrm{bc}}$ & $64.4^{\mathrm{bc}}$ & $55^{b}$ & $52.5^{\text {bc }}$ & $53.8^{b}$ & $53.5^{\text {bc }}$ \\
\hline $\begin{array}{l}\text { B. thuringiensis } \\
+320 \mathrm{~Gy}\end{array}$ & $40^{\mathrm{a}}$ & $49^{\mathrm{ab}}$ & $44.5^{\mathrm{ab}}$ & $66.6^{\mathrm{b}}$ & $75^{\mathrm{ab}}$ & $70.8^{\mathrm{ab}}$ & $69.9^{\mathrm{a}}$ & $60^{\mathrm{ab}}$ & $64.95^{\mathrm{a}}$ & $60.1^{\mathrm{ab}}$ \\
\hline $\begin{array}{l}\text { B. thuringiensis } \\
+640 \mathrm{~Gy}\end{array}$ & $44^{\mathrm{a}}$ & $55^{\mathrm{a}}$ & $49.5^{\mathrm{a}}$ & $72.2^{\mathrm{ab}}$ & $79^{\mathrm{a}}$ & $75.6^{\mathrm{a}}$ & $75^{a}$ & $65^{\mathrm{a}}$ & $70^{\mathrm{a}}$ & $65.03^{a}$ \\
\hline Azadirachtin & $25^{\mathrm{bc}}$ & $45^{b}$ & $35^{c}$ & $52.5^{c}$ & $62.5^{c}$ & $57.5^{c}$ & $47.1^{\mathrm{c}}$ & $47.5^{c}$ & $47.3^{c}$ & $46.6^{c}$ \\
\hline $\begin{array}{l}\text { B. thuringiensis } \\
\text { +Azadirachtin }\end{array}$ & $28^{\mathrm{b}}$ & $46.6^{b}$ & $37.3^{\text {bc }}$ & $55^{c}$ & $66.6^{\mathrm{bc}}$ & $60.8^{c}$ & $50^{\mathrm{bc}}$ & $50^{c}$ & $50^{\mathrm{bc}}$ & $49.4^{c}$ \\
\hline Diflubenzuron & $44^{a}$ & $55^{\mathrm{a}}$ & $49.5^{\mathrm{a}}$ & $75^{a}$ & $79^{a}$ & $77^{\mathrm{a}}$ & $76^{a}$ & $65^{a}$ & $70.5^{a}$ & $65.7^{\mathrm{a}}$ \\
\hline L.S.D ${ }_{0.05}$ & 6.11 & 7.39 & 7.54 & 6.19 & 8.39 & 8.08 & 7.26 & 7.54 & 6.11 & 8.21 \\
\hline \multicolumn{11}{|c|}{ Hydraulic Matabi sprayer (56L.IFed.) } \\
\hline B. thuringiensis & $18^{\mathrm{e}}$ & $22^{c}$ & $20^{\mathrm{d}}$ & $21^{\mathrm{c}}$ & $29^{c}$ & $25^{\mathrm{d}}$ & $7.7^{f}$ & $7.7^{\mathrm{d}}$ & $7.7^{\mathrm{d}}$ & $17.6^{\mathrm{e}}$ \\
\hline $\begin{array}{l}\text { B. thuringiensis } \\
+160 \mathrm{~Gy}\end{array}$ & $30^{\text {cd }}$ & $40^{\mathrm{b}}$ & $35^{\text {bc }}$ & $53^{b}$ & $66.6^{\mathrm{ab}}$ & $59.8^{\mathrm{abc}}$ & $50^{\text {cd }}$ & $48.8^{\mathrm{a}}$ & $49.4^{\mathrm{ab}}$ & 48.1 $1^{\text {bc }}$ \\
\hline $\begin{array}{l}\text { B. thuringiensis } \\
+320 \mathrm{~Gy}\end{array}$ & $35^{\mathrm{bc}}$ & $44^{\mathrm{ab}}$ & $39.5^{\mathrm{ab}}$ & $55^{b}$ & $69.9^{\mathrm{a}}$ & $62.5^{\mathrm{abc}}$ & $55^{\mathrm{bc}}$ & $50^{\mathrm{a}}$ & $52.5^{a}$ & $51.5^{\mathrm{b}}$ \\
\hline $\begin{array}{l}\text { B. thuringiensis } \\
+640 \mathrm{~Gy}\end{array}$ & $40^{\mathrm{ab}}$ & $49^{\mathrm{a}}$ & $44.5^{\mathrm{a}}$ & $62.2^{\mathrm{a}}$ & $70^{\mathrm{a}}$ & $66.1^{\mathrm{ab}}$ & $60^{\mathrm{ab}}$ & $50^{\mathrm{a}}$ & $55^{\mathrm{a}}$ & $55.2^{\mathrm{a}}$ \\
\hline Azadirachtin & $24^{\mathrm{de}}$ & $38^{\mathrm{b}}$ & $31^{\mathrm{c}}$ & $49^{b}$ & $59^{b}$ & $54^{c}$ & $42^{\mathrm{e}}$ & $40^{c}$ & $41^{c}$ & $42^{d}$ \\
\hline $\begin{array}{l}\text { B. thuringiensis } \\
\text { +Azadirachtin }\end{array}$ & $25^{\mathrm{d}}$ & $40^{\mathrm{b}}$ & $32.5^{c}$ & $52^{b}$ & $62.5^{\mathrm{ab}}$ & $57.3^{\text {bc }}$ & $45^{\mathrm{de}}$ & $44.4^{\mathrm{b}}$ & $44.7^{\mathrm{bc}}$ & $44.8^{\mathrm{cd}}$ \\
\hline Diflubenzuron & $42^{\mathrm{a}}$ & $49^{a}$ & $45.5^{\mathrm{a}}$ & $65^{a}$ & $70^{a}$ & $67.5^{a}$ & $62.2^{\mathrm{a}}$ & $50^{a}$ & $56.1^{\mathrm{a}}$ & $56.4^{\mathrm{a}}$ \\
\hline L.S.D D $_{0.05}$ & 6.11 & 7.29 & 6.37 & 6.19 & 8.39 & 8.43 & 6.28 & 7.03 & 6.37 & 3.47 \\
\hline
\end{tabular}

Numbers followed by the same letter at the same column are not significantly different at $P=0.05$. 
Table 9: Percent reduction in cottonseed bug populations during application by using two spraying machines with some compounds during the cotton season 2019.

\begin{tabular}{|c|c|c|c|c|c|c|c|c|c|c|}
\hline \multirow{3}{*}{ Compounds } & \multicolumn{9}{|c|}{ \% Reduction of cotton seed bug populations during application } & \multirow{3}{*}{$\begin{array}{l}\text { Seasonal } \\
\text { Average }\end{array}$} \\
\hline & \multicolumn{3}{|c|}{$1^{\text {st }}$ spray } & \multicolumn{3}{|c|}{$2^{\text {nd }}$ spray } & \multicolumn{3}{|c|}{$3^{\text {rd }}$ spray } & \\
\hline & 7 & 14 & Aver. & 7 & 14 & Aver. & 7 & 14 & Aver. & \\
\hline \multicolumn{11}{|c|}{ Pneumatic motor sprayer (Cifarilli) $(20$ L./fed.) } \\
\hline B. thuringiensis & $25^{\mathrm{c}}$ & $30^{\mathrm{d}}$ & $27.5^{\mathrm{e}}$ & $35^{\mathrm{e}}$ & $40^{\mathrm{d}}$ & $37.5^{\mathrm{d}}$ & $14 \mathrm{e}$ & $12^{\mathrm{d}}$ & $13^{\mathrm{e}}$ & $26^{d}$ \\
\hline $\begin{array}{l}\text { B. thuringiensis } \\
+160 \mathrm{~Gy}\end{array}$ & $42.2^{b}$ & $52.2^{\mathrm{bc}}$ & $47.2^{\mathrm{cd}}$ & $65^{\mathrm{bc}}$ & $66.6^{\mathrm{bc}}$ & $65.8^{\mathrm{b}}$ & $72.2^{\mathrm{bc}}$ & $70^{\mathrm{b}}$ & $71.1^{b}$ & $61.4^{b}$ \\
\hline $\begin{array}{l}\text { B. thuringiensis } \\
+320 \mathrm{~Gy}\end{array}$ & $50^{\mathrm{a}}$ & $56^{b}$ & $5^{\mathrm{bc}}$ & $69.9^{\mathrm{ab}}$ & $72.2^{\mathrm{ab}}$ & $71.1^{\mathrm{ab}}$ & $76^{\mathrm{ab}}$ & $70^{b}$ & $73^{b}$ & $65.7^{\mathrm{ab}}$ \\
\hline $\begin{array}{l}\text { B. thuringiensis } \\
+640 \mathrm{~Gy}\end{array}$ & $55^{\mathrm{a}}$ & $66.6^{\mathrm{a}}$ & $60.8^{\mathrm{ab}}$ & $73.3^{\mathrm{a}}$ & $79^{\mathrm{a}}$ & 76.2 $2^{\mathrm{a}}$ & $83^{\mathrm{a}}$ & $80^{\mathrm{a}}$ & $81.5^{\mathrm{a}}$ & $72.8^{\mathrm{a}}$ \\
\hline Azadirachtin & $35^{\mathrm{b}}$ & $45^{\mathrm{c}}$ & $40^{\mathrm{d}}$ & $55^{\mathrm{d}}$ & $58^{\mathrm{c}}$ & $56.5^{\mathrm{c}}$ & $62.2^{\mathrm{d}}$ & $45^{\mathrm{c}}$ & $53.6^{\mathrm{d}}$ & $50.03^{\mathrm{c}}$ \\
\hline $\begin{array}{l}\text { B. thuringiensis } \\
\text { +Azadirachtin }\end{array}$ & $38^{\mathrm{b}}$ & $49^{\mathrm{bc}}$ & $43.5^{d}$ & $62.2^{c}$ & $65^{\text {bc }}$ & $63.6^{b c}$ & $66.6^{\mathrm{cd}}$ & $64.4^{\mathrm{b}}$ & $65.5^{c}$ & $57.5^{\mathrm{bc}}$ \\
\hline Diflubenzuron & $55^{\mathrm{a}}$ & $70^{\mathrm{a}}$ & $62.5^{\mathrm{a}}$ & $73.3^{\mathrm{a}}$ & $79^{\mathrm{a}}$ & $76.2^{\mathrm{a}}$ & $84^{\mathrm{a}}$ & $80^{\mathrm{a}}$ & $82^{\mathrm{a}}$ & $73.6^{\mathrm{a}}$ \\
\hline L.S.D 0.05 & 7.54 & 8.39 & 8.53 & 6.24 & 8.95 & 7.78 & 7.72 & 7.26 & 3.32 & 7.89 \\
\hline \multicolumn{11}{|c|}{ Hydraulic Matabi sprayer (56L.IFed.) } \\
\hline B. thuringiensis & $24^{\mathrm{d}}$ & $27 \mathrm{e}$ & $25.5^{\mathrm{e}}$ & $29^{d}$ & $33^{d}$ & $31^{\mathrm{d}}$ & $10^{\mathrm{e}}$ & $7.7^{\mathrm{e}}$ & $8.85^{\mathrm{e}}$ & $21.8^{\mathrm{e}}$ \\
\hline $\begin{array}{l}\text { B. thuringiensis } \\
+160 \mathrm{~Gy}\end{array}$ & $38^{\mathrm{b}}$ & $48.8^{\mathrm{bc}}$ & $43.4^{b c}$ & $55^{b}$ & $58.8^{\mathrm{ab}}$ & $56.9^{\mathrm{ab}}$ & $62.2^{\mathrm{bc}}$ & $60^{b}$ & $61.1^{\mathrm{bc}}$ & $53.8^{\mathrm{bc}}$ \\
\hline $\begin{array}{l}\text { B. thuringiensis } \\
+320 \mathrm{~Gy}\end{array}$ & $40^{\mathrm{ab}}$ & $52.2^{\mathrm{ab}}$ & 46.1 $1^{\mathrm{bcc}}$ & $58^{\mathrm{ab}}$ & $62.2^{\mathrm{ab}}$ & $60.1^{\mathrm{ab}}$ & $69.9^{\mathrm{ab}}$ & $62.2^{\mathrm{ab}}$ & $66.1^{\mathrm{ab}}$ & $57.4^{\mathrm{ab}}$ \\
\hline $\begin{array}{l}\text { B. thuringiensis } \\
+640 \mathrm{~Gy}\end{array}$ & $45^{\mathrm{a}}$ & $56^{\mathrm{ab}}$ & $50.5^{\mathrm{ab}}$ & $62.2^{\mathrm{a}}$ & $65^{a}$ & $63.6^{\mathrm{a}}$ & $72.2^{\mathrm{a}}$ & $70^{\mathrm{a}}$ & $71.1^{\mathrm{a}}$ & $61.7^{\mathrm{ab}}$ \\
\hline Azadirachtin & $30^{\text {cd }}$ & $39^{d}$ & $34.5^{\mathrm{d}}$ & $44^{\mathrm{c}}$ & $49^{\mathrm{c}}$ & $46.5^{\mathrm{c}}$ & $53^{\mathrm{d}}$ & $40^{d}$ & $46.5^{d}$ & $42.5^{\mathrm{d}}$ \\
\hline $\begin{array}{l}\text { B. thuringiensis } \\
\text { +Azadirachtin }\end{array}$ & $35^{\mathrm{bc}}$ & $42.2^{\mathrm{cd}}$ & $38.6^{\mathrm{cd}}$ & $52.2^{\mathrm{b}}$ & $55^{\mathrm{bc}}$ & $53.6^{\text {bc }}$ & $58.8^{\mathrm{cd}}$ & $50^{c}$ & $54.4^{\text {cd }}$ & $48.9^{\mathrm{cd}}$ \\
\hline Diflubenzuron & $45^{\mathrm{a}}$ & $60^{\mathrm{a}}$ & $52.5^{\mathrm{a}}$ & $62.2^{\mathrm{a}}$ & $65^{\mathrm{a}}$ & $6^{63.6^{\mathrm{a}}}$ & $73^{a}$ & $70^{\mathrm{a}}$ & $71.5^{\mathrm{a}}$ & $62.4^{\mathrm{a}}$ \\
\hline L.S.D ${ }_{0.05}$ & 6.16 & 8.68 & 8.26 & 6.22 & 7.54 & 7.47 & 7.72 & 7.54 & 8.003 & 7.87 \\
\hline
\end{tabular}

Numbers followed by the same letter at the same column are not significantly different at $P=0.05$.

Table 10: Percent reduction in cottonseed bug infestations during application by using two spraying machines with some compounds during cotton season 2018.

\begin{tabular}{|c|c|c|c|c|c|c|c|c|c|c|}
\hline \multirow{3}{*}{ Compounds } & \multicolumn{9}{|c|}{$\%$ Reduction of cotton seed bug infestations during application } & \multirow{3}{*}{$\begin{array}{l}\text { Seasonal } \\
\text { Average }\end{array}$} \\
\hline & \multicolumn{3}{|c|}{$1^{\text {st }}$ spray } & \multicolumn{3}{|c|}{$2^{\text {nd }}$ spray } & \multicolumn{3}{|c|}{$3^{\text {rd }}$ spray } & \\
\hline & 7 & 14 & Aver. & 7 & 14 & Aver. & 7 & 14 & Aver. & \\
\hline \multicolumn{11}{|c|}{ Pneumatic motor sprayer (Cifarilli) $(20$ L./fed.) } \\
\hline B. thuringiensis & $25^{\mathrm{d}}$ & $40^{\mathrm{d}}$ & $32.5^{\mathrm{e}}$ & $50^{\mathrm{b}}$ & $25^{c}$ & $37.5^{\mathrm{d}}$ & $20^{\mathrm{e}}$ & $7.7^{\mathrm{d}}$ & $13.9^{\mathrm{e}}$ & $27.9^{\mathrm{e}}$ \\
\hline $\begin{array}{l}\text { B. thuringiensis } \\
+160 \mathrm{~Gy}\end{array}$ & $38^{\mathrm{bc}}$ & $58.8^{\mathrm{ab}}$ & $48.4^{\text {bc }}$ & $65^{\mathrm{a}}$ & $66.6^{b}$ & $65.8^{\mathrm{b}}$ & $75^{\mathrm{c}}$ & $71^{\mathrm{b}}$ & $73^{c}$ & $62.4^{\text {bc }}$ \\
\hline $\begin{array}{l}\text { B. thuringiensis } \\
+320 \mathrm{~Gy}\end{array}$ & $42.2^{\mathrm{ab}}$ & $62.2^{\mathrm{a}}$ & $52.2^{\mathrm{ab}}$ & $69.9^{\mathrm{a}}$ & $79^{\mathrm{a}}$ & $74.5^{\mathrm{a}}$ & $82^{b}$ & $75^{b}$ & $78.5^{\text {bc }}$ & $68.4^{\mathrm{ab}}$ \\
\hline $\begin{array}{l}\text { B. thuringiensis } \\
+640 \mathrm{~Gy}\end{array}$ & $50^{\mathrm{a}}$ & $65^{\mathrm{a}}$ & $57.5^{\mathrm{a}}$ & $72.2^{\mathrm{a}}$ & $82.2^{\mathrm{a}}$ & $77.2^{\mathrm{a}}$ & $89^{\mathrm{a}}$ & $85^{\mathrm{a}}$ & $87^{\mathrm{ab}}$ & $73.9^{\mathrm{a}}$ \\
\hline Azadirachtin & $25^{d}$ & $50^{c}$ & $37.5^{\text {de }}$ & $50^{\mathrm{b}}$ & $61.3^{\mathrm{b}}$ & $55.7^{\mathrm{c}}$ & $65.4^{\mathrm{d}}$ & $50^{c}$ & $57.7^{d}$ & $50.3^{d}$ \\
\hline $\begin{array}{l}\text { B. thuringiensis } \\
\text { +Azadirachtin }\end{array}$ & $32^{\mathrm{cd}}$ & $54^{\mathrm{bc}}$ & $43^{\mathrm{cd}}$ & $55^{\mathrm{b}}$ & $66.6^{b}$ & $60.8^{\mathrm{bc}}$ & $72^{c}$ & $70^{\mathrm{b}}$ & $71^{\mathrm{c}}$ & $58.3^{\mathrm{cd}}$ \\
\hline Diflubenzuron & $50^{\mathrm{a}}$ & $66.6^{\mathrm{a}}$ & $58.3^{\mathrm{a}}$ & $73^{\mathrm{a}}$ & $85^{\mathrm{a}}$ & $79^{\mathrm{a}}$ & $90^{\mathrm{a}}$ & $86^{\mathrm{a}}$ & $88^{\mathrm{a}}$ & $75.1^{\mathrm{a}}$ \\
\hline L.S.D 0.05 & 7.72 & 7.54 & 8.53 & 7.54 & 8.88 & 7.29 & 6.32 & 8.62 & 8.74 & 8.53 \\
\hline \multicolumn{11}{|c|}{ Hydraulic Matabi sprayer (56L..Fed.) } \\
\hline B. thuringiensis & $22^{\mathrm{d}}$ & $38^{\mathrm{d}}$ & $30^{c}$ & $40^{\mathrm{cd}}$ & $19^{\mathrm{e}}$ & $29.5^{\mathrm{d}}$ & $15^{\mathrm{d}}$ & $7.7^{\mathrm{d}}$ & $11.4^{\mathrm{d}}$ & $23.6^{\mathrm{e}}$ \\
\hline $\begin{array}{l}\text { B. thuringiensis } \\
+160 \mathrm{~Gy}\end{array}$ & $30^{\mathrm{c}}$ & $45^{\mathrm{abcd}}$ & $37.5^{b c}$ & $55^{\mathrm{b}}$ & $59.9^{\mathrm{bc}}$ & $57.5^{\mathrm{b}}$ & $65^{b c}$ & $62.2^{b}$ & $63.6^{b}$ & $52.9^{\mathrm{bc}}$ \\
\hline $\begin{array}{l}\text { B. thuringiensis } \\
+320 \mathrm{~Gy}\end{array}$ & $38^{\mathrm{b}}$ & $48^{\mathrm{abc}}$ & $43^{\mathrm{ab}}$ & $59^{a b}$ & $66.6^{\mathrm{ab}}$ & $62.8^{\mathrm{ab}}$ & $69.9^{\mathrm{ab}}$ & $65^{\mathrm{ab}}$ & $67.5^{\mathrm{ab}}$ & $57.8^{\mathrm{ab}}$ \\
\hline $\begin{array}{l}\text { B. thuringiensis } \\
+640 \mathrm{~Gy}\end{array}$ & $45^{\mathrm{a}}$ & $50^{a b}$ & $47.5^{a}$ & $62.2^{\mathrm{ab}}$ & $70^{\mathrm{a}}$ & $66.1^{\mathrm{ab}}$ & $75^{\mathrm{a}}$ & $72^{a}$ & $73.5^{\mathrm{a}}$ & $62.4^{\mathrm{a}}$ \\
\hline Azadirachtin & $23^{d}$ & $40^{\text {cd }}$ & $31.5^{\mathrm{c}}$ & $37^{d}$ & $50^{d}$ & $43.5^{c}$ & $59^{\mathrm{c}}$ & $40^{c}$ & $49.5^{c}$ & $41.5^{\mathrm{d}}$ \\
\hline $\begin{array}{l}\text { B. thuringiensis } \\
\text { +Azadirachtin }\end{array}$ & $25^{\mathrm{cd}}$ & $42.2^{\mathrm{bcd}}$ & $33.6^{c}$ & $45^{c}$ & $52.2^{\mathrm{cd}}$ & $48.6^{c}$ & $62.2^{\mathrm{bc}}$ & $60^{b}$ & $61.1^{\mathrm{b}}$ & $47.8^{\mathrm{cd}}$ \\
\hline Diflubenzuron & $45^{\mathrm{a}}$ & $52.2^{\mathrm{a}}$ & $48.6^{\mathrm{a}}$ & $65^{\mathrm{a}}$ & $70^{\mathrm{a}}$ & $67.5^{\mathrm{a}}$ & $77^{\mathrm{a}}$ & $72^{\mathrm{a}}$ & $74.5^{\mathrm{a}}$ & $63.5^{\mathrm{a}}$ \\
\hline L.S.D D. $_{05}$ & 6.16 & 7.54 & 7.78 & 7.54 & 8.95 & 8.53 & 7.54 & 7.72 & 7.78 & 7.95 \\
\hline
\end{tabular}

Numbers followed by the same letter at the same column are not significantly different at $P=0.05$. 
Table 11: Percent reduction in cottonseed bug infestations during application by using two spraying equipment with some compounds during the cotton season 2019.

\begin{tabular}{|c|c|c|c|c|c|c|c|c|c|c|}
\hline \multirow{3}{*}{ Compounds } & \multicolumn{9}{|c|}{$\%$ Reduction of cotton seed bug infestations during application } & \multirow{3}{*}{$\begin{array}{l}\text { Seasonal } \\
\text { Average }\end{array}$} \\
\hline & \multicolumn{3}{|c|}{$1^{\text {st }}$ spray } & \multicolumn{3}{|c|}{$2^{\text {nd }}$ spray } & \multicolumn{3}{|c|}{$3^{\text {rd }}$ spray } & \\
\hline & 7 & 14 & Aver. & 7 & 14 & Aver. & 7 & 14 & Aver. & \\
\hline \multicolumn{11}{|c|}{ Pneumatic motor sprayer (Cifarilli) (20 L./fed.) } \\
\hline B. thuringiensis & $25^{\mathrm{c}}$ & $30^{\mathrm{d}}$ & $27.5^{\mathrm{d}}$ & $40^{\mathrm{e}}$ & $45^{\mathrm{e}}$ & $42.5^{\mathrm{d}}$ & $40^{\mathrm{d}}$ & $30^{\mathrm{c}}$ & $35^{d}$ & $35^{d}$ \\
\hline $\begin{array}{l}\text { B. thuringiensis } \\
+160 \mathrm{~Gy}\end{array}$ & $52^{b}$ & $62.2^{\mathrm{b}}$ & $57.1^{b c}$ & $72.2^{\mathrm{bcd}}$ & $75^{\mathrm{c}}$ & $73.6^{b}$ & $72^{\mathrm{ab}}$ & $69^{b}$ & $70.5^{b}$ & $67.1^{b}$ \\
\hline $\begin{array}{l}\text { B. thuringiensis } \\
+320 \mathrm{~Gy}\end{array}$ & $62.2^{\mathrm{a}}$ & $65^{b}$ & $63.6^{\mathrm{ab}}$ & $77^{\mathrm{abc}}$ & $79^{\mathrm{bc}}$ & $78^{\mathrm{ab}}$ & $77^{\mathrm{ab}}$ & $70^{\mathrm{b}}$ & $73.5^{\mathrm{ab}}$ & $71.7^{a b}$ \\
\hline $\begin{array}{l}\text { B. thuringiensis } \\
+640 \mathrm{~Gy}\end{array}$ & $67^{\mathrm{a}}$ & $75^{\mathrm{a}}$ & $71^{\mathrm{a}}$ & $80^{\mathrm{ab}}$ & $85^{\mathrm{ab}}$ & $82.5^{\mathrm{a}}$ & $80^{\mathrm{a}}$ & $82^{a}$ & $81^{\mathrm{a}}$ & $78.2^{\mathrm{a}}$ \\
\hline Azadirachtin & $45^{\mathrm{b}}$ & $55^{\mathrm{c}}$ & $50^{\mathrm{c}}$ & $65^{\mathrm{d}}$ & $60^{\mathrm{d}}$ & $62.5^{\mathrm{c}}$ & $55^{\mathrm{c}}$ & $35^{\mathrm{c}}$ & $45^{\mathrm{c}}$ & $52.5^{\mathrm{c}}$ \\
\hline $\begin{array}{l}\text { B. thuringiensis } \\
\text { +Azadirachtin }\end{array}$ & $49^{\mathrm{b}}$ & $59^{\mathrm{bc}}$ & $54^{c}$ & $69^{\mathrm{cd}}$ & $72.2^{c}$ & $70.6^{b}$ & $70^{b}$ & $65^{b}$ & $67.5^{b}$ & $64.03^{b}$ \\
\hline Diflubenzuron & $67^{\mathrm{a}}$ & $75^{\mathrm{a}}$ & $71^{\mathrm{a}}$ & $82^{a}$ & $88^{\mathrm{a}}$ & $85^{a}$ & $80^{\mathrm{a}}$ & $80^{\mathrm{a}}$ & $80^{\mathrm{a}}$ & $78.7^{\mathrm{a}}$ \\
\hline L.S.D $\mathbf{D}_{0.05}$ & 7.72 & 6.28 & 8.21 & 8.39 & 8.003 & 7.72 & 8.39 & 8.88 & 7.78 & 8.88 \\
\hline \multicolumn{11}{|c|}{ Hydraulic Matabi sprayer (56L.IFed.) } \\
\hline B. thuringiensis & $22^{\mathrm{b}}$ & $28^{\mathrm{d}}$ & $25^{d}$ & $35^{\mathrm{c}}$ & $39^{\mathrm{e}}$ & $37^{\mathrm{e}}$ & $30^{\mathrm{d}}$ & $19^{\mathrm{e}}$ & $24.5^{d}$ & $28.8^{\mathrm{e}}$ \\
\hline $\begin{array}{l}\text { B. thuringiensis } \\
+160 \mathrm{~Gy}\end{array}$ & $46^{\mathrm{a}}$ & $54^{\mathrm{bc}}$ & $50^{\mathrm{abc}}$ & $65^{\mathrm{ab}}$ & $66.6^{\text {bc }}$ & $65.8^{\mathrm{bc}}$ & $65^{\mathrm{ab}}$ & $58^{\mathrm{bc}}$ & $61.5^{\mathrm{ab}}$ & $59.1^{\text {bc }}$ \\
\hline $\begin{array}{l}\text { B. thuringiensis } \\
+320 \mathrm{~Gy}\end{array}$ & $50^{\mathrm{a}}$ & $58^{\mathrm{abc}}$ & $54^{\mathrm{abc}}$ & $69^{\mathrm{ab}}$ & $72.2^{\mathrm{abc}}$ & $70.6^{\mathrm{abc}}$ & $70^{\mathrm{ab}}$ & $65^{\mathrm{ab}}$ & $67.5^{\mathrm{ab}}$ & $64.03^{\mathrm{abc}}$ \\
\hline $\begin{array}{l}\text { B. thuringiensis } \\
+640 \mathrm{~Gy}\end{array}$ & $52^{\mathrm{a}}$ & $62.2^{\mathrm{ab}}$ & $57.1^{\mathrm{ab}}$ & $72^{\mathrm{a}}$ & $75^{\mathrm{ab}}$ & $73.5^{\mathrm{ab}}$ & $72.2^{\mathrm{a}}$ & $70^{\mathrm{a}}$ & $71.1^{\mathrm{a}}$ & $67.2 \mathrm{ab}$ \\
\hline Azadirachtin & $44^{\mathrm{a}}$ & $50^{\mathrm{c}}$ & $47^{\mathrm{c}}$ & $61^{b}$ & $50^{\mathrm{d}}$ & $55.5^{\mathrm{d}}$ & $46.6^{\mathrm{c}}$ & $30^{\mathrm{d}}$ & $38.3^{c}$ & $46.9^{d}$ \\
\hline $\begin{array}{l}\text { B. thuringiensis } \\
\text { +Azadirachtin }\end{array}$ & $45^{\mathrm{a}}$ & $52^{\circ}$ & $48.5^{b c}$ & $62.2^{\mathrm{b}}$ & $64.4^{c}$ & $63.3^{\mathrm{c}}$ & $62.2^{b}$ & $55^{\mathrm{c}}$ & $58.6^{b}$ & $56.8^{\mathrm{c}}$ \\
\hline Diflubenzuron & $52^{\mathrm{a}}$ & $65^{\mathrm{a}}$ & $58.5^{\mathrm{a}}$ & $73^{a}$ & $79^{\mathrm{a}}$ & $76^{\mathrm{a}}$ & $72.2^{\mathrm{a}}$ & $70^{\mathrm{a}}$ & $71.1^{\mathrm{a}}$ & $68.5^{\mathrm{a}}$ \\
\hline L.S.D . $_{0.05}$ & 8.43 & 8.07 & 8.58 & 7.89 & 8.39 & 7.39 & 8.39 & 8.003 & 9.06 & 8.58 \\
\hline
\end{tabular}

Numbers followed by the same letter at the same column are not significantly different at $P=0.05$.

\section{Cotton Crop Parameters:}

The parameters of cotton crop (seed numbers, lint and seed weights) for each 100 opened cotton bolls was an important step to clear the effective of seven treatments used on the quality of cotton crop as illustrated in Tables $(12 \& 13)$ that mentioned the role of gamma radiation treatments for potentiating $B$. thuringiensis used to purpose of crop quality enhancement. Also, utilizing pneumatic motor sprayer (Cifarilli) $(20$ L./Fed.) enhanced the application comparing with the economy Hand-held Hydraulic sprayer (Matabi) (56 L./fed.) uses.

\section{a. Seed Numbers:}

B. thuringiensis +640 Gy treatment caused increasing in cottonseed numbers to 1345 $\& 1255$ and $1275 \& 1098$ seeds/ opened 100 bolls during application by Pneumatic motor sprayer (Cifarilli) (20 L./Fed.) and Hand-held Hydraulic sprayer (Matabi) (56 L./fed.) in 2018 \& 2019 cotton seasons compared to untreated $989 \& 954.5$ seeds/ 100 opened boll at 2018 \& 2019 cotton seasons, respectively. Also, B. thuringiensis +320 Gy had increased the cotton seed compared to untreated, followed by B. thuringiensis $+160 \mathrm{~Gy}$ that exposed to gamma radiation doses. Also, B. thuringiensis + azadirachtin treatment caused seed number increasing compared to $B$. thuringiensis or azadirachtin when applied singly.

\section{b. Lint Weight (g):}

B. thuringiensis +640 Gy had the highest lint weight/100 opened boll, it was $99 \& 98$ and $91 \& 89 \mathrm{~g} / 100$ opened boll for Penumatic motor sprayer (Cifarilli) (20 L./Fed.) and Hand-held Hydraulic sprayer (Matabi) (56 L./fed.) during cotton seasons 2018 \& 2019, respectively compared with untreated $(50.2 \& 46.4 \mathrm{~g})$ for cotton seasons $2018 \& 2019$. While $B$. thuringiensis without exposure to gamma doses had the least value comparing with other treatments used. 


\section{c.Seed Weight (g):}

B. thuringiensis $+640 \mathrm{~Gy}$ had the highest cotton seed weights with two spraying machine used comparing with $86.6 \& 755 \mathrm{~g}$ for untreated cotton seeds at cotton seasons 2018 and 2019 (Tables 12 \& 13), Followed by B. thuringiensis +320 Gy, B. thuringiensis +160 Gy, B. thuringiensis + azadirachtin, diflubenzuron, azadirachtin and $B$. thuringiensis , respectively .

It could be classified the seven treatments used efficacies against three cotton boll pests (P. gossypiella, E. insulana and O. hyalinipennis) on the field application to four categories as follows:

1.The first category that had a high efficacy on tested pests than other treatments. It's were diflubenzuron and B. thuringiensis $+640 \mathrm{~Gy}$.

2.The second category that had a high efficacy on tested pests but slightly decreased compared with the first category. It's were B. thuringiensis $+320 \mathrm{~Gy}$ and B. thuringiensis + 160 Gy.

3.The third category that had intermediate efficacy on tested pests. It's were $B$. thuringiensis + azadirachtin and azadirachtin singly.

4.The fourth category had lower efficacy on tested pests. It's was B. thuringiensis when used singly.

In general, the Pneumatic motor sprayer (Cifarilli) (20 L./fed.) gave the best application for treatments than Hydraulic Matabi sprayer (56L.IFed.) of two cotton seasons because the degree of homogeneity of droplets spectrum in the case of Pneumatic motor sprayer (Cifarilli) is higher than handheld Hydrulic (Matabi) sprayer.

Table 12: Cotton crop parameters as affected by some compound's applications using two ground equipment during the cotton season 2018

\begin{tabular}{|c|c|c|c|c|c|c|}
\hline \multirow[b]{2}{*}{ Compounds } & \multicolumn{6}{|c|}{ Average weights (gm/100boll) } \\
\hline & $\begin{array}{c}\text { Seed } \\
\text { numbers }\end{array}$ & $\begin{array}{c}\text { Comparison } \\
\text { With } \\
\text { untreated }\end{array}$ & $\begin{array}{c}\text { Lint } \\
\text { weights }\end{array}$ & $\begin{array}{c}\text { Comparison } \\
\text { With } \\
\text { untreated } \\
\end{array}$ & $\begin{array}{c}\text { Seed } \\
\text { weights }\end{array}$ & $\begin{array}{c}\text { Comparison } \\
\text { With } \\
\text { untreated }\end{array}$ \\
\hline \multicolumn{7}{|c|}{ Pneumatic motor sprayer (Cifarilli) $(20$ L./fed.) } \\
\hline Untreated & $989^{c}$ & $-^{d}$ & $50.2^{e}$ & $-f$ & $86.6^{\mathrm{e}}$ & $-f$ \\
\hline B. thuringiensis & $1100^{\mathrm{bc}}$ & $+111^{\mathrm{c}}$ & $65^{d}$ & $+14.8^{e}$ & $95^{\mathrm{e}}$ & $+8.4^{\mathrm{e}}$ \\
\hline $\begin{array}{l}\text { B. thuringiensis } \\
+160 \mathrm{~Gy}\end{array}$ & $1310^{\mathrm{a}}$ & $+321 \mathrm{ab}$ & $89 \mathrm{ab}$ & $+38.8^{\mathrm{abc}}$ & $118^{\mathrm{abc}}$ & $+31.4^{b}$ \\
\hline $\begin{array}{l}\text { B. thuringiensis } \\
+320 \mathrm{~Gy}\end{array}$ & $1320^{\mathrm{a}}$ & $+331^{\mathrm{a}}$ & $92^{\mathrm{ab}}$ & $+41.8^{\mathrm{ab}}$ & $122^{\mathrm{ab}}$ & $+35.4^{b}$ \\
\hline $\begin{array}{l}\text { B. thuringiensis } \\
+640 \mathrm{~Gy}\end{array}$ & $1345^{\mathrm{a}}$ & $+356^{\mathrm{a}}$ & $99^{\mathrm{a}}$ & $+48.8^{\mathrm{a}}$ & $130^{\mathrm{a}}$ & $+43.4^{\mathrm{a}}$ \\
\hline Azadirachtin & $1220^{\mathrm{ab}}$ & $+231^{b}$ & $70^{\text {cd }}$ & $+19.8^{\text {de }}$ & 97 de & $+10.4^{\mathrm{e}}$ \\
\hline $\begin{array}{l}\text { B. thuringiensis } \\
\text { +Azadirachtin }\end{array}$ & $1304^{\mathrm{a}}$ & $+315^{\mathrm{ab}}$ & $84^{b}$ & $+33.8^{\mathrm{bc}}$ & $112^{\text {bcd }}$ & $+25.4^{c}$ \\
\hline Diflubenzuron & $1290^{\mathrm{a}}$ & $+301 \mathrm{ab}$ & $79^{\text {bc }}$ & $+28.8^{\mathrm{cd}}$ & $102^{\text {cde }}$ & $+15.4^{\mathrm{d}}$ \\
\hline L.S.D D.05 $_{1}$ & 137.5 & 87.5 & 12.7 & 11.05 & 15.3 & 4.93 \\
\hline \multicolumn{7}{|c|}{ Hydraulic Matabi sprayer (56L.|Fed.) } \\
\hline Untreated & $989^{b}$ & $-d$ & $50.2^{d}$ & $-d$ & $86.6^{b}$ & $-\mathrm{e}$ \\
\hline B. thuringiensis & $1000^{\mathrm{b}}$ & $+11^{d}$ & $54^{d}$ & $+3.8^{\mathrm{d}}$ & $87^{b}$ & $+0.4^{\mathrm{e}}$ \\
\hline $\begin{array}{l}\text { B. thuringiensis } \\
+160 \mathrm{~Gy}\end{array}$ & $1220^{\mathrm{a}}$ & $+231^{a b}$ & $80^{a b}$ & $+29.8^{b c}$ & $102^{a b}$ & $+15.4^{c}$ \\
\hline $\begin{array}{l}\text { B. thuringiensis } \\
+320 \mathrm{~Gy}\end{array}$ & $1260^{\mathrm{a}}$ & $+271^{a b}$ & $85^{a b}$ & $+34.8^{\mathrm{ab}}$ & $112^{\mathrm{a}}$ & $+25.4^{b}$ \\
\hline $\begin{array}{l}\text { B. thuringiensis } \\
+640 \mathrm{~Gy}\end{array}$ & $1275^{a}$ & $+286^{\mathrm{a}}$ & $91^{\mathrm{a}}$ & $+40.8^{\mathrm{a}}$ & $118^{\mathrm{a}}$ & $+31.4^{\mathrm{a}}$ \\
\hline Azadirachtin & $1080^{b}$ & $+91^{c}$ & $60^{\text {cd }}$ & $+9.8^{d}$ & $89^{b}$ & $+2.4^{\mathrm{e}}$ \\
\hline $\begin{array}{l}\text { B. thuringiensis } \\
\text { +Azadirachtin }\end{array}$ & $1210^{\mathrm{a}}$ & $+221^{a b}$ & $78^{a b}$ & $+27.8^{b c}$ & $95^{b}$ & $+8.4^{\mathrm{d}}$ \\
\hline Diflubenzuron & $1200^{\mathrm{a}}$ & $+211^{b}$ & $72^{\mathrm{bc}}$ & $+21.8^{c}$ & $90^{\mathrm{b}}$ & $+3.4^{\mathrm{e}}$ \\
\hline L.S.D $D_{0.05}$ & 107.2 & 62.1 & 12.9 & 9.554 & 15.3 & 4.58 \\
\hline
\end{tabular}

Numbers followed by the same letter at the same column are not significantly different at $P=0.05$. 
Table 13: Cotton crop parameters as affected by some compounds applications using two ground sprayer machines during the cotton season 2019.

\begin{tabular}{|c|c|c|c|c|c|c|}
\hline \multirow[b]{2}{*}{ Compounds } & \multicolumn{6}{|c|}{ Average weights (gm/100boll) } \\
\hline & $\begin{array}{c}\text { Seed } \\
\text { numbers }\end{array}$ & $\begin{array}{c}\text { Comparison } \\
\text { With } \\
\text { untreated }\end{array}$ & $\begin{array}{c}\text { Lint } \\
\text { weights }\end{array}$ & $\begin{array}{c}\text { Comparison } \\
\text { With } \\
\text { untreated }\end{array}$ & $\begin{array}{c}\text { Seed } \\
\text { weights }\end{array}$ & $\begin{array}{c}\text { Comparison } \\
\text { With } \\
\text { untreated }\end{array}$ \\
\hline \multicolumn{7}{|c|}{ Pneumatic motor sprayer (Cifarilli) $(20$ L./fed.) } \\
\hline Untreated & $954.5^{c}$ & $-e$ & $46.4^{\mathrm{d}}$ & $-\mathrm{g}$ & $75.5^{\mathrm{e}}$ & $-\mathrm{e}$ \\
\hline B. thuringiensis & $990^{c}$ & $+35.5^{d}$ & $51^{d}$ & $+4.6^{f}$ & $82^{\text {de }}$ & $+6.5^{\mathrm{de}}$ \\
\hline $\begin{array}{c}\text { B. thuringiensis } \\
+160 \mathrm{~Gy}\end{array}$ & $1240^{\mathrm{a}}$ & $+285.5^{\mathrm{a}}$ & $80^{\mathrm{bc}}$ & $+33.6^{c}$ & $103^{b c}$ & $+27.5^{b c}$ \\
\hline $\begin{array}{l}\text { B. thuringiensis } \\
+320 \mathrm{~Gy}\end{array}$ & $1245^{a}$ & $+290.5^{\mathrm{a}}$ & $89^{\mathrm{ab}}$ & $+42.6^{\mathrm{b}}$ & $112^{\mathrm{ab}}$ & $+36.5^{a b}$ \\
\hline $\begin{array}{l}\text { B. thuringiensis } \\
+640 \mathrm{~Gy}\end{array}$ & $1255^{\mathrm{a}}$ & $+300.5^{\mathrm{a}}$ & $98^{\mathrm{a}}$ & $+51.6^{\mathrm{a}}$ & $120^{\mathrm{a}}$ & $+44.5^{\mathrm{a}}$ \\
\hline Azadirachtin & $1030^{\mathrm{bc}}$ & $+75.5^{\mathrm{c}}$ & $55^{d}$ & $+8.6^{\mathrm{e}}$ & 84 de & +8.5 de \\
\hline $\begin{array}{l}\text { B. thuringiensis } \\
+ \text { Azadirachtin }\end{array}$ & $1200^{\mathrm{ab}}$ & $+245.5^{b}$ & $71^{c}$ & $+24.6^{\mathrm{d}}$ & $93 \mathrm{~cd}$ & $+17.5 \mathrm{~cd}$ \\
\hline Diflubenzuron & $1190^{\mathrm{ab}}$ & $+235.5^{b}$ & $68^{c}$ & $+21.6^{\mathrm{d}}$ & $90^{\text {cde }}$ & $+14.5^{\mathrm{d}}$ \\
\hline L.S.D ${ }_{0.05}$ & 174.5 & 33.2 & 11.5 & 3.82 & 15.5 & 11.3 \\
\hline \multicolumn{7}{|c|}{ Hydraulic Matabi sprayer (56L.(Fed.) } \\
\hline Untreated & $954.5^{c}$ & $-f$ & $46.4^{\mathrm{d}}$ & $-{ }^{\mathrm{e}}$ & $75.5^{\mathrm{d}}$ & $-e$ \\
\hline B. thuringiensis & $985^{\mathrm{c}}$ & $+30.5^{\mathrm{e}}$ & $49^{d}$ & $+2.6^{\mathrm{e}}$ & $78^{\mathrm{d}}$ & $+2.5^{\mathrm{e}}$ \\
\hline $\begin{array}{l}\text { B. thuringiensis } \\
+160 \mathrm{~Gy}\end{array}$ & $1086^{\mathrm{a}}$ & $+131.5^{b}$ & $77^{\mathrm{b}}$ & $+30.6^{b}$ & $95^{\mathrm{bc}}$ & $+19.5^{b c}$ \\
\hline $\begin{array}{l}\text { B. thuringiensis } \\
+320 \mathrm{~Gy}\end{array}$ & $1090^{\mathrm{a}}$ & $+135.5^{\mathrm{ab}}$ & $82^{\mathrm{ab}}$ & $+35.6^{b}$ & $104^{\mathrm{ab}}$ & $+28.5^{\mathrm{ab}}$ \\
\hline $\begin{array}{l}\text { B. thuringiensis } \\
+640 \mathrm{~Gy}\end{array}$ & $1098^{a}$ & $+143.5^{\mathrm{a}}$ & $89^{a b}$ & $+42.6^{\mathrm{a}}$ & $111^{\mathrm{a}}$ & $+35.5^{\mathrm{a}}$ \\
\hline Azadirachtin & $1000^{\text {bc }}$ & $+45.5^{\mathrm{d}}$ & $52^{d}$ & $+5.6^{\mathrm{e}}$ & $80^{d}$ & +4.5 de \\
\hline $\begin{array}{l}\text { B. thuringiensis } \\
+ \text { Azadirachtin }\end{array}$ & $1080^{\mathrm{a}}$ & $+125.5^{b}$ & $69^{c}$ & $+22.6^{c}$ & $90^{\mathrm{bcd}}$ & $+14.5^{\mathrm{cd}}$ \\
\hline Diflubenzuron & $1065^{\mathrm{ab}}$ & $+110.5^{c}$ & $63^{c}$ & $+16.6^{\mathrm{d}}$ & $85^{\mathrm{cd}}$ & $+9.5^{\text {cde }}$ \\
\hline L.S.D ${ }_{0.05}$ & 69.6 & 10.8 & 7.93 & 5.61 & 13.5 & 9.62 \\
\hline
\end{tabular}

Numbers followed by the same letter at the same column are not significantly different at $P=0.05$.

\section{Spray Coverage on Cotton Leaves of Insecticides Used:}

Data in Table (14) showed that, B. thuringiensis, B. thuringiensis $+160 \mathrm{~Gy}, B$. thuringiensis +320 Gy, B. thuringiensis +640 Gy, Azadirachtin, B. thuringiensis +Azadirachtin, and Diflubenzuron using two ground spraying equipment and varied spraying volumes depending on the sprayer used. Data indicated that in general a satisfactory coverage was obtained on cotton plants. The spectrum of droplets ranging between 122-185 $\mu \mathrm{m}$ (VMD) i.e. more than 50 droplets/ $\mathrm{cm}^{2}$, with sufficient number ranging from $33-253 \mathrm{~N} / \mathrm{cm}^{2}$. a-Penumatic Motor Sprayer (Cifarilli) (20 L/Fed.):

Data in Table (15) showed that the lost spray percentages which were $10.2,9,8.9,8.7$, $9.9,9.3 \& 8.5 \%$ from the total spray volume in the case of recommended dose of $B$. thuringiensis, B. thuringiensis $+160 \mathrm{~Gy}$, B. thuringiensis $+320 \mathrm{~Gy}$, B. thuringiensis +640 Gy, Azadirachtin, B. thuringiensis +Azadirachtin, and Diflubenzuron , respectively. b-Hydraulic Matabi Sprayer (56L/fed.):

Data in Table (15) showed that the lost spray percentages which were $16.5,15,14.8,14.4,16$, 15.4 and $14 \%$ from the total spray volume in the case of recommended dose of $B$. thuringiensis, B. thuringiensis $+160 \mathrm{~Gy}$, B. thuringiensis $+320 \mathrm{~Gy}$, B. thuringiensis +640 Gy, Azadirachtin, B. thuringiensis +Azadirachtin, and Diflubenzuron, respectively. 
Table 14: Spraying coverage on cotton plants and the ground produced by certain ground spraying equipment during seasons 2018 and 2019 at Qalubiya Governorate.

\begin{tabular}{|c|c|c|c|c|c|c|}
\hline \multirow{3}{*}{ Compounds } & \multicolumn{6}{|c|}{$\begin{array}{l}\text { Pneumatic motor sprayer (Cifarilli) } \\
(20 \text { L/Fed.) }\end{array}$} \\
\hline & \multicolumn{2}{|c|}{ Upper level } & \multicolumn{2}{|c|}{ Middle level } & \multicolumn{2}{|c|}{ Lower level } \\
\hline & $\mathrm{N} / \mathrm{cm}^{2}$ & $\mathrm{VMD}$ & $\underset{2}{\mathrm{~N} / \mathrm{cm}}$ & $\mathrm{VMD}$ & $\mathrm{N} / \mathrm{cm}^{2}$ & $\mathrm{VMD}$ \\
\hline B. thuringiensis & $118^{\mathrm{e}}$ & $159^{\mathrm{a}}$ & $113^{\mathrm{b}}$ & $160^{\mathrm{a}}$ & $109^{f}$ & $162^{\mathrm{a}}$ \\
\hline B. thuringiensis $+160 \mathrm{~Gy}$ & $150^{\mathrm{d}}$ & $138^{\mathrm{c}}$ & $148^{\mathrm{b}}$ & $150^{\mathrm{b}}$ & $140^{\mathrm{d}}$ & $150^{c}$ \\
\hline B. thuringiensis $+320 \mathrm{~Gy}$ & $170^{c}$ & $135^{\mathrm{c}}$ & $165^{\mathrm{ab}}$ & $138^{\mathrm{c}}$ & $155^{\mathrm{c}}$ & $140^{\mathrm{d}}$ \\
\hline B. thuringiensis $+640 \mathrm{~Gy}$ & $197^{\mathrm{b}}$ & $131^{\mathrm{d}}$ & $185^{\mathrm{a}}$ & $135^{c}$ & $175^{b}$ & $138^{\mathrm{d}}$ \\
\hline Azadirachtin & $120^{\mathrm{e}}$ & $153^{\mathrm{b}}$ & $115^{\mathrm{b}}$ & $150^{\mathrm{b}}$ & $110^{f}$ & $155^{b}$ \\
\hline B. thuringiensis +Azadirachtin & $122^{\mathrm{e}}$ & $130^{\text {de }}$ & $120^{\mathrm{b}}$ & $135^{c}$ & $115^{\mathrm{e}}$ & $152^{\mathrm{bc}}$ \\
\hline Diflubenzuron & $253^{\mathrm{a}}$ & $127^{\mathrm{e}}$ & $220^{\mathrm{ab}}$ & $130^{\mathrm{d}}$ & $200^{\mathrm{a}}$ & $128^{\mathrm{e}}$ \\
\hline \multirow[t]{2}{*}{ L.S.D ${ }_{0.05}$} & 3.76 & 3.384 & 116.5 & 3.317 & 3.621 & 3.225 \\
\hline & \multicolumn{6}{|c|}{ Hydraulic Matabi sprayer (56L.IFed.) } \\
\hline B. thuringiensis & $109^{f}$ & $185^{\mathrm{a}}$ & $120^{\mathrm{g}}$ & $180^{\mathrm{a}}$ & $105^{f}$ & $170^{\mathrm{a}}$ \\
\hline B. thuringiensis $+160 \mathrm{~Gy}$ & $153^{\mathrm{d}}$ & $153^{\mathrm{b}}$ & $160^{\mathrm{d}}$ & $155^{\mathrm{c}}$ & $140^{\mathrm{d}}$ & $157^{\mathrm{bc}}$ \\
\hline B. thuringiensis $+320 \mathrm{~Gy}$ & $160^{c}$ & $150^{\mathrm{b}}$ & $173^{c}$ & $152^{\mathrm{d}}$ & $148^{\mathrm{c}}$ & $155^{\mathrm{c}}$ \\
\hline B. thuringiensis $+640 \mathrm{~Gy}$ & $170^{\mathrm{b}}$ & $135^{\mathrm{c}}$ & $180^{\mathrm{b}}$ & $138^{\mathrm{e}}$ & $160^{\mathrm{b}}$ & $138^{\mathrm{d}}$ \\
\hline Azadirachtin & $110^{f}$ & $155^{\mathrm{b}}$ & $125^{f}$ & $159^{\mathrm{b}}$ & $108^{f}$ & $160^{\mathrm{b}}$ \\
\hline B. thuringiensis + Azadirachtin & $136^{\mathrm{e}}$ & $154^{\mathrm{b}}$ & $150^{\mathrm{e}}$ & $156^{\mathrm{c}}$ & $130^{\mathrm{e}}$ & $159^{\mathrm{b}}$ \\
\hline Diflubenzuron & $186^{\mathrm{a}}$ & $122^{\mathrm{d}}$ & $195^{\mathrm{a}}$ & $125^{f}$ & $170^{\mathrm{a}}$ & $128^{\mathrm{e}}$ \\
\hline L.S.D ${ }_{0.05}$ & 3.154 & 5.294 & 3.154 & 1.779 & 3.154 & 2.866 \\
\hline
\end{tabular}

L.S.D. at $(0.5 \%)$ between treatments. Numbers followed by the same letter at the same column are not significantly different.

Table 15: Spray lost between cotton plants produced by certain ground spraying equipment during seasons 2018 and 2019 at Qalubiya Governorate.

\begin{tabular}{|c|c|c|c|c|c|c|c|c|c|c|c|c|c|c|}
\hline \multirow{3}{*}{$\begin{array}{c}\text { Equipment } \\
\text { Treatments }\end{array}$} & \multicolumn{14}{|c|}{ Pneumatic motor sprayer (Cifarilli) (20 L./fed.) } \\
\hline & \multicolumn{2}{|c|}{$\begin{array}{c}B . \\
\text { thuringiensis }\end{array}$} & \multicolumn{2}{|c|}{$\begin{array}{l}\text { B. thuringiensis } \\
+160 \mathrm{~Gy}\end{array}$} & \multicolumn{2}{|c|}{$\begin{array}{l}\text { B. thuringiensis } \\
+320 \mathrm{~Gy}\end{array}$} & \multicolumn{2}{|c|}{$\begin{array}{l}\text { B. thuringiensis } \\
+640 \mathrm{~Gy}\end{array}$} & \multicolumn{2}{|c|}{ Azadirachtin } & \multicolumn{2}{|c|}{$\begin{array}{l}\text { B. thuringiensis } \\
\text { +Azadirachtin }\end{array}$} & \multicolumn{2}{|c|}{ Diflubenzuron } \\
\hline & $\mathrm{N} / \mathrm{cm}^{2}$ & VMD & $\mathrm{N} / \mathrm{cm}^{2}$ & $\mathrm{VMD}$ & $\mathrm{N} / \mathrm{cm}^{2}$ & VMD & $\mathrm{N} / \mathrm{cm}^{2}$ & $\mathrm{VMD}$ & $\mathrm{N} / \mathrm{cm}^{2}$ & $\mathrm{VMD}$ & $\mathrm{N} / \mathrm{cm}^{2}$ & $\mathrm{VMD}$ & $\mathrm{N} / \mathrm{cm}^{2}$ & $\mathrm{VMD}$ \\
\hline $\begin{array}{c}\text { Mean On } \\
\text { plants }\end{array}$ & 113 & 160 & 146 & 146 & 163 & 137 & 186 & 134 & 115 & 153 & 119 & 139 & 224 & 128 \\
\hline Ground & 35 & 170 & 39 & 132 & 44 & 164 & 48 & 135 & 34 & 160 & 33 & 157 & 57 & 130 \\
\hline $\begin{array}{c}\% \mathrm{~N} / \mathrm{Cm}^{2} \text { on } \\
\text { ground (spray } \\
\text { lost }\end{array}$ & 10.2 & - & 9 & - & 8.9 & - & 8.7 & - & 9.9 & - & 9.3 & - & 8.5 & - \\
\hline Equipment & \multicolumn{14}{|c|}{ Hydraulic Matabi sprayer (56L.IFed.) } \\
\hline Treatments & \multicolumn{2}{|c|}{$\begin{array}{c}B . \\
\text { thuringiensis }\end{array}$} & \multicolumn{2}{|c|}{$\begin{array}{l}\text { B. thuringiensis } \\
+160 \mathrm{~Gy}\end{array}$} & \multicolumn{2}{|c|}{$\begin{array}{l}\text { B. thuringiensis } \\
\quad+320 \mathrm{~Gy}\end{array}$} & \multicolumn{2}{|c|}{$\begin{array}{l}\text { B. thuringiensis } \\
+640 \mathrm{~Gy}\end{array}$} & \multicolumn{2}{|c|}{ Azadirachtin } & \multicolumn{2}{|c|}{$\begin{array}{l}\text { B. thuringiensis } \\
\text { +Azadirachtin }\end{array}$} & \multicolumn{2}{|c|}{ Diflubenzuron } \\
\hline & $\begin{array}{c}\mathrm{N} / \mathrm{cm} \\
2\end{array}$ & VMD & $\mathrm{N} / \mathrm{cm}^{2}$ & VMD & $\mathrm{N} / \mathrm{cm}^{2}$ & VMD & $\mathrm{N} / \mathrm{cm}^{2}$ & VMD & $\mathrm{N} / \mathrm{cm}^{2}$ & $\mathrm{VMD}$ & $\mathrm{N} / \mathrm{cm}^{2}$ & $\mathrm{VMD}$ & $\mathrm{N} / \mathrm{cm}^{2}$ & VMD \\
\hline $\begin{array}{c}\text { Mean On } \\
\text { plants }\end{array}$ & 111 & 178 & 151 & 155 & 160 & 152 & 170 & 137 & 114 & 158 & 139 & 156 & 184 & 125 \\
\hline ON ground & 55 & 185 & 68 & $14 !$ & 71 & 146 & 73 & 148 & 55 & 1775 & 64 & 1700 & 77 & 150 \\
\hline $\begin{array}{c}\% \mathrm{~N} / \mathrm{Cm}^{2} \text { on } \\
\text { ground (spray } \\
\text { lost }\end{array}$ & 16.5 & - & 15 & - & 14.8 & - & 14.4 & - & 16 & - & 15.4 & - & 14 & - \\
\hline
\end{tabular}

\section{Relations Between Spray Quality and The General Reduction Percentages of Two} Seasons by Using Certain Bioinsecticides Applied on Cotton Plants:

Data in Table (16) showed that homogeneity of spray coverage was high and in the case of Pneumatic motor sprayer (Cifarilli) (20 L./fed.) followed by Hydraulic Matabi sprayer (56L/fed.).The difference in the mortality percentages was due to the different modes of action of the bio-insecticides, degree of gamma radiation, the different spray volume used. 
and the degree of homogeneity of the droplets spectrum in the case of Knapsack motor sprayer (Cifarilli) is higher than the handheld Hydrulic (Matabi) sprayer.

Also, there was no Phytotoxic effect on Cotton leaves after application treatments with pesticides in all treatments there was no change in the leaves color, and no leaf curling or flaming up phenomena has happened in case of all treatments.

The rate of performance of Pneumatic motor sprayer (Cifarilli) (20 L./fed.), the sprayer was 12 Fed./day. It was the best equipment, but the lowest rate of performance was Hydraulic Matabi sprayer (56L/fed.) since it could spray only 3.45 Fed./day.

Table 16: Relationship between field spray quality of Bio-insecticides by Penumatic motor sprayer (Cifarilli) (20 L/Fed.) and Hydraulic Matabi sprayer (56L.IFed.) during seasons 2018 and 2019 at Qalubiya Governorate.

\begin{tabular}{|c|c|c|c|c|c|c|}
\hline \multirow{3}{*}{ Treatments } & \multicolumn{6}{|c|}{ Spray quality $=\mathrm{VMD} / \mathrm{N} / \mathrm{cm}^{2}=$ degree of homogeneity } \\
\hline & \multicolumn{3}{|c|}{$\begin{array}{l}\text { Pneumatic motor sprayer } \\
\text { (Cifarilli) (20 L./fed.) }\end{array}$} & \multicolumn{3}{|c|}{$\begin{array}{c}\text { Hydraulic Matabi sprayer } \\
\text { (56L.).Fed.) }\end{array}$} \\
\hline & $\begin{array}{c}\text { Upper } \\
\text { level }\end{array}$ & $\begin{array}{c}\text { Middle } \\
\text { level }\end{array}$ & $\begin{array}{c}\text { Lower } \\
\text { level }\end{array}$ & $\begin{array}{c}\text { Upper } \\
\text { level }\end{array}$ & $\begin{array}{c}\text { Middle } \\
\text { level }\end{array}$ & $\begin{array}{c}\text { Lower } \\
\text { level }\end{array}$ \\
\hline B. thuringiensis & $1.3^{\mathrm{a}}$ & $1.4^{\mathrm{a}}$ & $1.49^{\mathrm{a}}$ & $1.7^{\mathrm{a}}$ & $1.51^{\mathrm{a}}$ & $1.61^{\mathrm{a}}$ \\
\hline $\begin{array}{l}\text { B. thuringiensis } \\
+160 \mathrm{~Gy}\end{array}$ & $0.92^{\mathrm{a}}$ & $1.01^{\mathrm{a}}$ & $1.07^{\mathrm{a}}$ & $1^{\mathrm{ab}}$ & $0.97^{\mathrm{a}}$ & $1.12^{\mathrm{a}}$ \\
\hline $\begin{array}{l}\text { B. thuringiensis } \\
+320 \mathrm{~Gy}\end{array}$ & $0.79^{\mathrm{a}}$ & $0.84^{\mathrm{a}}$ & $0.9^{\mathrm{a}}$ & $0.94^{\mathrm{ab}}$ & $0.88^{\mathrm{a}}$ & $1.05^{\mathrm{a}}$ \\
\hline $\begin{array}{l}\text { B. thuringiensis } \\
+640 \mathrm{~Gy}\end{array}$ & $0.66^{\mathrm{a}}$ & $0.73^{\mathrm{a}}$ & $0.79^{\mathrm{a}}$ & $0.79 \mathrm{ab}$ & $0.77^{\mathrm{a}}$ & $0.86^{\mathrm{a}}$ \\
\hline Azadirachtin & $1.28^{\mathrm{a}}$ & $1.3^{\mathrm{a}}$ & $1.4^{\mathrm{a}}$ & $1.4^{\mathrm{ab}}$ & $1.27^{\mathrm{a}}$ & $1.48^{\mathrm{a}}$ \\
\hline $\begin{array}{l}\text { B. thuringiensis } \\
+ \text { Azadirachtin }\end{array}$ & $1.07^{\mathrm{a}}$ & $1.1^{\mathrm{a}}$ & $1.3^{\mathrm{a}}$ & $1.1^{\mathrm{ab}}$ & $1.04^{\mathrm{a}}$ & $1.2^{\mathrm{a}}$ \\
\hline Diflubenzuron & $0.5^{\mathrm{a}}$ & $0.59^{\mathrm{a}}$ & $0.64^{a}$ & $0.66^{b}$ & $0.64^{\mathrm{a}}$ & $0.75^{a}$ \\
\hline L.S.D D. $05_{1}$ & 0.818 & 0.853 & 0.846 & 0.833 & 0.834 & 0.939 \\
\hline
\end{tabular}

S.Q. $=$ spray quality. $=\mathrm{VMD} / \mathrm{N} / \mathrm{cm}^{2}=$ Spray quality $($ degree of homogeneity).

The spray height is constant $\sim 0.5$ meter in all treatments

$\mathrm{VMD}=$ Volume mean diameter, $\mathrm{N} / \mathrm{cm}^{2}=$ Number of droplets $/ \mathrm{cm}^{2}$

Numbers followed by the same letter at the same column are not significantly different at $P=0.05$.

Gamma radiation doses contribute to enhancement from efficacies of $B$. thuringiensis when exposed to gamma radiation doses of $160,320 \& 640$ Gy against three cotton boll pests of $P$. gossypiella, E. insulana, and O.hyalinipennis. Previous works agreed with the current study as Amer (2006) showed that the combination of gamma irradiation with Dipel $2 x$ activated the spores of the biocide compound and caused a potentiation effect. Also, Amer (2006) carried out the field experiments during the two cotton seasons 2004 and 2005. The results showed that the efficiency of Dipel-2x increased gradually with gamma irradiation from 5 to $80 \mathrm{~Gy}$. Also, the treatments increased lint and seed weights (gm/100bolls). Amer, et al (2012) mentioned that LC50's on subjected insects ( $P$. gossypiella, S. littoralis, and A. craccivora) treated with $B$. thuringiensis and exposed to gamma doses (150, $250 \& 350 \mathrm{~Gy})$ were lower than unexposing B. thuringiensis to gamma doses. Amer, et al. (2015a) exposed $B$. thuringiensis, $M$. anisopliae and biopolymer compound (chitosan) to gamma doses of 15 , $30 \& 60 \mathrm{~Gy}$, respectively for potentiating effect. It showed potentiated effect especially with a dose of 60 Gy was more effective than other doses used against $S$. littoralis treated as $4^{\text {th }}$ instar larvae at different efficiencies tests. Amer, et al. (2015b) mentioned that $B$. thuringiensis $+60 \mathrm{~Gy}$ lead to swelling of the outer cuticle fibrous layer of S. littoralis larvae integument. Furthermore, hypodermis layer had distention and damage in S. littoralis larvae. Also, it occurrence of split and destruction of muscles into small portions and remarkable suffered on the fat body cells as vacuolization and destroyed the fat body membranous sheath; in addition to alterations influences in the midgut of $S$. littoralis as destroyed of 
columnar or hyperphesia cells padding midgut, damage of brush border with excess of goblet cells. Furthermore, Amer, et al. (2018) reported that a heavily \% DNA of S. littoralis had destruction rang: 40-92\% caused by Chitosan +60 Gy that had the highly \% DNA destruction $(8.399 \%)$, followed by chitosan $+30 \mathrm{~Gy}(7.829 \%)$, M. anisopliae +15 Gy $(5.681 \%)$, chitosan $(3.991 \%)$, B. thuringiensis +30 Gy (3.902\%), M. anisopliae+ 60 Gy $(2.604 \%)$ and chitosan +15 Gy $(1.868 \%)$. Amer, et al. (2019) stated that gamma-ray doses $(50 \& 500 \mathrm{~Gy})$ treatments were the most efficacy against $E$. insulana egg stage than magnetic flux treatments $(20 \& 180 \mathrm{mlt})$.

Diflubenzuron compound as Insect Development Inhibitor makes a disturbance of formation of lamellate deposition of the procuticle. After fixation and staing, the globular material could be found instead. The new instar was either not able to ecdyse due to lack of rigidity of its exoskeleton or dies shortly after ecdysis. The ovicidal activity caused either by contact activity on egg or adult female treatment. The larvae in the egg had fully developed but unable to leave the egg (Wright and Retanakaran, 1987). In addition, Srinivasan and Uthanasamy (2001) conducted the experiment was conducted to evaluate the efficacy of diflubenzuron alone and in combination with profenofos and $\alpha$-cypermethrin against the American bollworm, $H$. armigera. The combinations were found to reduce the incidence of $H$. armigera. Moreover, Mahmoud, et al. (2014) evaluated the toxicity of lufenuron and diflubenzuron against the newly hatched larvae of $P$. gossypiella under laboratory conditions. There is prolongation in larval and pupal development in diflubenzuron than lufenuron. In contrast was happened in the adult stage; high reduction in total eggs, hatchability\% and longevity.

At current work, the additive compound of B. thuringiensis + azadirachtin had potentiating effect than $B$. thuringiensis or azadirachtin singly. Meanwhile, Rafiq et al. (2012) conducted that azadirachtin extracts in different parts of plants showed significant mortality response against $3^{\text {rd }}$ instar larvae of cotton $P$. gossypiella, $S$. litura and $H$. armigera. The surviving insects showed the behavior with a decrease in insect weight and slower feeding activity as compared to the control. The efficacy of these extracts may be further enhanced by using $1^{\text {st }}$ and $2^{\text {nd }}$ instar larvae of these insects in bioassays as well as optimizing dose concentration and treatment time. Also, Dawkar, et al. (2019) suggested that azadrachtin targets more than one protein in $H$. armigera and hence could be a potent biopesticide.

For evaluation the field performance of Low-Volume spraying machines; Pneumatic motor sprayer (Cifarilli) (20 L/Fed.) and Hydrulic Matabi sprayer (56L/fed.), respectively ; to spray B. thuringiensis, B. thuringiensis $+160 \mathrm{~Gy}$, B. thuringiensis $+320 \mathrm{~Gy}$, B. thuringiensis + 640 Gy, Azadirachtin, B. thuringiensis +Azadirachtin, and Diflubenzuron using two ground spraying equipment and varied spraying with total recommended dose. A satisfactory coverage was obtained on cotton plants, the droplet spectrum was obtained in field experiment was agreed with the optimum droplet sizes mentioned by (Mathews,1992), in case of low volume equipment. It could be recommended to use these compounds with LV spraying equipment with not less than (20 L./Fed.). Also, the best equipment in this respect was Pneumatic motor sprayer (Cifarilli) (20 L/Fed.) followed Hand-held Hydraulic sprayer (Matabi) (56 L.Ifed.), the tested equipment under study. The rate of performance of Pneumatic motor sprayer (Cifarilli) (20 L./fed.), the sprayer was 12 Fed./day. It was the best equipment, but the lowest rate of performance was Hand-held Hydraulic sprayer (Matabi) (56 L.lfed.) since it could spray only $3.45 \mathrm{Fed}$./day. (18 L/fed.) droplets $/ \mathrm{cm}^{2}$ and the lost spray-on ground, and these results agreed with Hindy et al. (2004), Genidy et al. (2005) which recommended KZ oil and Pyriproxyfen followed by Agerin using low volume spraying because of reducing the time lost in process filling the machines, improve the homogeneity of the spray solution on the Cotton plant leaves and saving the lost spray on the ground, these results also in agreement with Bakr et al. (2014) they recommended by using Profenofos 
followed by Pyriproxyfen and Spinosad with Agromondo sprayer (20L/fed.). Dar et al. (2019) showed that Motorized Knapsack sprayer (Agromondo) (20 L.Fed.) was the best equipment to control seedling pests at the early season of Cotton. The rate of performance of Knapsack motor sprayer (Arimitsu) was 15.25 Fed./day. It was the best equipment, but the lowest rate of performance was Hand-held Hydraulic sprayer (Matabi) (56 L.Ifed.) since it could spray only 3.4 Fed./day. Also, the lowest spray volume, the lowest percentage $9.4 \%$ of lost spraying between plants occurred by Pneumatic motor sprayer (Cifarilli) (20 L/Fed.), this result was agreed with Hindy et al. (1997), who mentioned that there was a positive relationship between rate of application and lost spray-on ground. Also, the best equipment in this respect was Knapsack motor sprayer (Cifarilli) (20 L/Fed.) followed by Hand-held Hydraulic sprayer (Matabi) (56 L.Ifed.) the tested equipment under study, these results were in agreement with Dar et al. (2020). Spray Quality was near to 1 in case of Pneumatic motor sprayer (Cifarilli) (20 L/Fed.) and Hand-held Hydraulic sprayer (Matabi) (56 L.lfed.) which indicated high spray coverage homogeneity and best controlling of the three boll pests, this result were agreed with (Mathews,1992), Dobson (2001) whom illustrated the typical values for spray quality (homogeneity)=more than 2.5 (very poor) for Hydraulic nozzle, whereas $=2$ for air-shear nozzle.

\section{CONCLUSION}

Generally, it could be concluded that Pneumatic motor sprayer (Cifarilli) (20 L/Fed.) contribute to success more control than Hand-held Hydraulic sprayer (Matabi) (56 L.Ifed.) due to more homogenous spraying and a higher rate of performance.

It could be recommended to $B$. thuringiensis, $B$. thuringiensis $+160 \mathrm{~Gy}, B$. thuringiensis $+320 \mathrm{~Gy}$, B. thuringiensis $+640 \mathrm{~Gy}$, Azadirachtin, B. thuringiensis +Azadirachtin, and Diflubenzuron with low volume (LV) spraying equipment with not less than (20 L./fed.) which revealed successful results. There was a negative complete correlation between (VMD) and the reduction percentage of the three boll pests of Cotton while there was a positive complete correlation between $\mathrm{N} / \mathrm{cm}^{2}$ and the reduction percentage of the three boll pests of Cotton in all treatments. Moreover, gamma radiation doses (160, $320 \& 640 \mathrm{~Gy}$ ) improve the potentiating $B$. thuringiensis action to become the efficacy action was the highly if it compared with $B$. thuringiensis without exposing to gamma radiation doses or both of them used singly.

\section{REFERENCES}

Abedi, Z.; Saber, M.; Vojoudi, S.; Mahdavi, V. and Parsaeyan, E. (2014): Acute, sublethal, and combination effects of azadirachtin and Bacillus thuringiensis on the cotton bollworm, Helicoverpa armigera. journal of Insect Science, 14: 1-9.

Amer, R.A. (2006): Combination of gamma irradiation with Bacillus thuringiensis (Kurs.) and the synergistic effect of two bioinsecticide mixture for controlling the pink bollworm, Pectinophora gossypiella (Saund.) in cotton bolls. J.Egypt.Ger.Soc.Zool. 51: $1-13$.

Amer, R.A.; Ahmed, M.A. and Hatem, A.E. (2012): Effect of gamma irradiation combined with B.T. biocide treatments on some insect pests in laboratory. Egypt. J. Agric. Res., 90 (3): 1041-1053.

Amer, R.A.; Hassan, N.N.; Sheba, O.Sh. and Abdel-Salam, D.A. (2019a): Gamma radiation potency to potentiate some compounds for controlling three cotton boll pests. Egypt. Acad. J. Biolog. Sci., 11(3): 1-13.

Amer, R. A.; Kandil, M. A. and El- Shenawy, R. M. (2019b): Comparison between gamma rays and magnetic flux effects on biological and life table assays of Earias insulan (Boisd.) eggs. Egypt. Acad. J. Biolog. Sci., 12(3):121-131. 
Amer, R.A.; Salem, M.S.; Abdel-Salam, D. and Hassan, N.N. (2018): Comet assay parameters of Spodoptera littoralis (Boisd.) (Noctuidae: Lepidoptera) larvae resistance system cells as affected by different compounds exposed to gamma irradiation. Egypt. J. Agric. Res.,96 (3): 885-907.

Amer, R.A.; Yacoub, Sh.S.; Nouh, G.M. and Hatem, A.E. (2015a): Gamma irradiation to potentiate some bio-agents' compounds against the cotton leaf worm, Spodoptera littoralis (Boisd.) (Lepidoptera: Noctuidae). Egyptian Journal Biological Pest Control, 25(2): 445-455.

Amer, R.A.; Yacoub, Sh. S. and Salem, M.S. (2015b): Histopathological effects of some bio agent compounds exposed to gamma irradiation on the cotton leaf worm, Spodoptera littoralis (Boisd.). J. Plant Prot. And Path., Mansoura Univ., 6(10): 1369-1380.

Ananthakrishan, T.N., Raman, K. and Sanjayan, K.P. (1982): Comparative growth rate, fecundity, and behavioral diversity of the dusky cotton bug, Oxycarenus hyalinipennis (Costa) (Hemiptera: Lygaeidae) on certain Malvaceous host plants. Proceeding of Indian Science Academy, 48: 577-584.

Badr, A.N.; El-Sisi, G.A. and Abdel Meguid, M.A. (1995): Evaluation of some locally formulated petroleum oils for controlling cotton leaf worm. J. Agric. Sci. Mansoura Univ., 20(5): 2557-2562.

Bakr, R. F.; Hindy, M.A., Ahmed, N. S., Genidy, N. A. and Dar, R. A. (2014): Field comparison between droplet distribution and the bioresidual activity of different insecticides against Spodoptera littoralis (Boisd) by using certain ground spraying equipment on cotton plants. J. Egypt. Acad. Soc. Biolo. Sci. 7(1):187-193.

Dar, R.A.; Moustafa, H.Z. and Salem, M.S. (2019): Field studies of different insecticides on cotton seedling pests and their natural enemies by using certain ground spraying equipment at Qalyopia governorate. International J. of Entomology Research. 4(4):132140.

Dar, R.A.; Lotfy, D.E. and Moustafa H.Z. (2020): Field application of bio-insecticides on spiny bollworm, Earias insulana (Bosid.) on cotton by using recent low volume ground spraying equipment. Egypt. Acad. J. Biolog. Sci. (A. Entomology) Vol. 13(1): 47-57.

Dawkar, V.V.; Barage, S.H.; Barbole, R.S.; Fatangare, A.; Grimalt, S.; Haldar, S.; Heckel, D.G.; Gupta, V.S.; Thulasiram, H.V.; Svatos, A. and Giri, A.P. (2019): Azadirachtin-A from Azadirachta indica impacts multiple biological targets in cotton bollworm, Helicoverpa armigera. ACS Omega, 4: 9531-9541.

Dobson, H.M. (2001): Desert locust Guidelines, Food and Agricultural Organization of the United Nations (FAO), Rome, $2^{\text {nd }}$ edition, part 4, Control, PP 85.

Dokic, D.; Stanisavljevic, R.; Marckvic, J.; Milenkovic, J; Terzic, D.; Vasic, S. and Barac, S. (2018): Performance testing of field crop sprayers in the Rasina District. Acta Agri. Serbica, 23 (45): 27-36.

Duncan, D.B. (1955): Multiple ranges and multiple F.test. Biomerics. 11:1-42.

Genidy, N.A.; Bakr, R. F.; Hindy, M. A. and Dar, R. A. (2005): Bioresidual activity certain insecticides against Spodoptera littoralis (Boisd) by using low volume ground spraying equipment on cotton plants. J. Egypt. Acad. Soc. Environ. Develop.,(AEntomolgy),6(1):1-21.

Henderson, C.F. and Tilton, E.W. (1955): Test with acaricides against the brown wheat mite. J. Econ. Ent., 48:157-161.

Hindy, M.A. (1989): Residual activity of certain insecticides as affected by aerial application parameters. Ph. D. Thesis. Fac. Ageric., Ain Shams Univ., Egypt, 177 pp.

Hindy, M.A. (1992): Qualitative distribution of watery dyed spray produced by certain ground sprayers in cotton. Bull. Ent. Soc., Egypt 19:221-227. 
Hindy, M.A.; El-Sayed, A.M.; Abd El-Salam, S.M. and Samy, M.A. (1997): Qualitative Assessment of certain insecticides applied by different ground sprayers against whitefly, Bemicia tabaci (Geen.) on eggplant. Egypt. J. Agric. Res., 75 (3): 565-577.

Hindy, M.A.; Bakr, R.F.; Genidy, N.A. and Dar, R.A. (2004): Qualitative distribution of certain insecticides deposits and artificial targets on the cotton leafworm larvae by using certain ground spraying equipment. on cotton plants.J. Egypt. Acad. Soc. Environ. Develop. (A. Entomology), 5(2): 91-112.

Khan, R.R.; Ahmed, S.; Saleem, M.W. and Nadeem, M. (2007): Field evaluation of different insecticides against spotted bollworms Earias spp. at district sahiwal. Pak. Entomol., 29(2): 129-134.

Magdoline, A. S.; Mohamed, K.E. and Safwat, H.Y. (1992): Less soil contamination with pesticides through modification and implemention of ground application techniques.Egypt.J.Appl.Sci.,7(7):157-170.

Mahmoud, M.A.; El-Sebai, O.A.; Naguib, S.M. and Abd-El Hamed, M.S. (2014): Toxicological and biological effects of lufenuron and diflubenzuron on pink bollworm, Pectinophora gossypiella (Saunders) (Lepidoptera: Gelechiidae). J. Plant Prot. And Path., Mansoura Univ., 5(1): 49-57.

Matthews, G.A. (1992): Pesticide application methods. $2^{\text {nd }}$ edition, Longman Harlow Publ., U.K.,405 PP.

Noble, L.W. (1969): Fifty years research on the pink bollworm in the United States agriculture. Handbook No. 357, Washington, D.C. 20402.

Rafiq, M.M.; Dahot, M.U.; Naqvi, S.H.; Mali, M. and Ali, N. (2012): Efficacy of neem (Azadirachta indica A. Juss) callus and cells suspension extracts against three lepidopteran insects of cotton. Journal of Medicinal Plants Research, 6(40): 5344-5349.

Sallam, H.A. and Ibrahim, S.M. (1993): Inherited sterility in progeny of gamma irradiated male cotton leaf worm, Spodoptera littoralis (Boisd.). Proceeding of the final research Co-ordination meeting. Phoenix, Arizona, 9-13 September.

Soon, L.G. (1986): Diamondback moth management (Proc. $1^{\text {st }}$ Int. Workshop), Asian Research and Development Center. Taipei: 159.

Srinivas, M. and Patil, B.V. (2004): Quantitative and qualitative loss caused by dusky cotton bug, Oxycarenus laetus Kirby on cotton. Karnataka Journal of Agriculture Sciences 17:487-490.

Srinivasan, R. and Uthamasamy, S. (2001): Bioefficacy of diflubenzuron, insecticides and diflubenzuron + insecticide mixtures on the incidence of American bollworm, Helicoverpa armigera (Hub.) in cotton. Pestology, xxv (11): 13-15.

Sweet, M.H. (2000): Seed and chinch bugs. Pages 143-264 in C.W. Schaefer and A.R. Panizzi, Heteroptera of Economic Importance. CRC, Boca Raton. 197-205 pp.

Wright, J.E. and Retanakaran, A. (1987): Chitin and benzophenyl ureas. 38:1-315, Dr. W. Junk publisher, series entomologica, editor K.A. Spencer; ISBN: 13:978-94-010-86387. 


\section{ARABIC SUMMARY}

التأثير التعاونى بين آثعة جاما وتوزيع قطيرات الرش لبعض المبيدات الحيوية لمكافحة ثلاث آفات على لوز القطن في مصر.

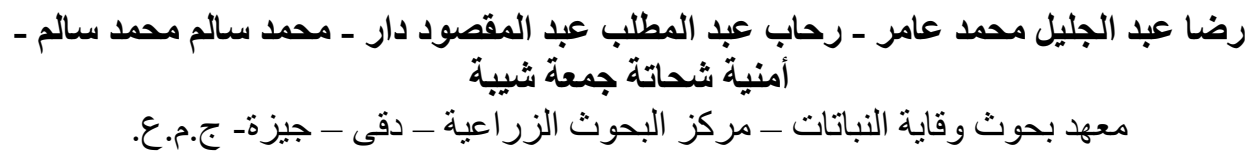

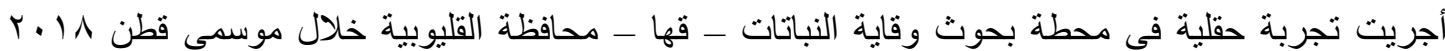

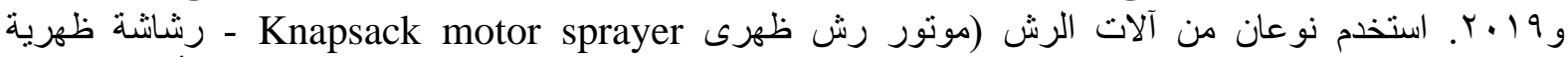
( Economy micron ulva sprayer

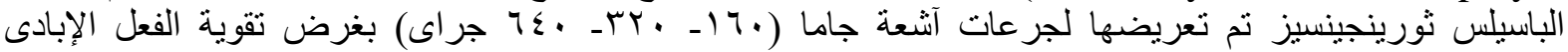

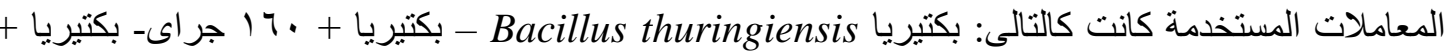
• r ج جراى- بكتيريا + • ع ج جر ای- بكتيريا + مستخلص الأزدر اكتين - مستخلص الأزدر اكتين و البكتيريا منفردين -

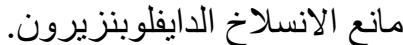

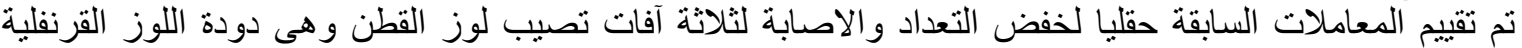
Oxycarenus ودودة اللوز الثوكية Earias insulana وبقة بذرة القطنة . hyalinipennis

عققت معاملات الرش بالموتور الظهرى نتائج أفضل من معاملات الرشاشة الظهرية. كما تعتبر معاملة البكتيريا

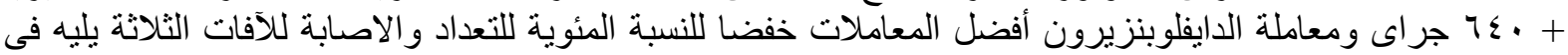

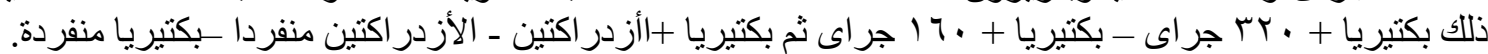

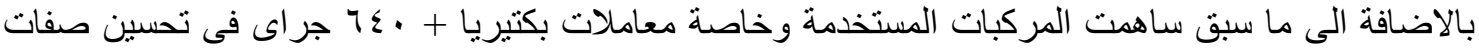

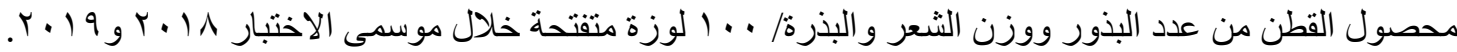

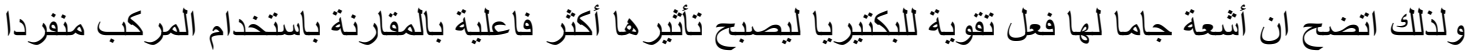

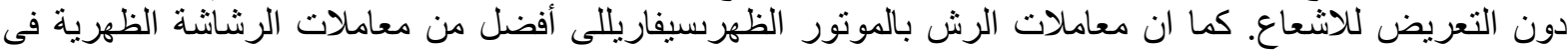

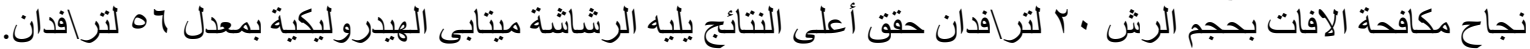

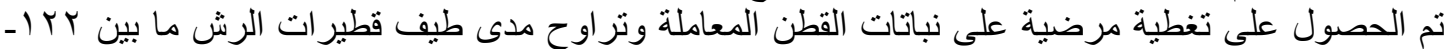

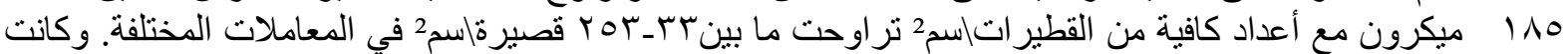

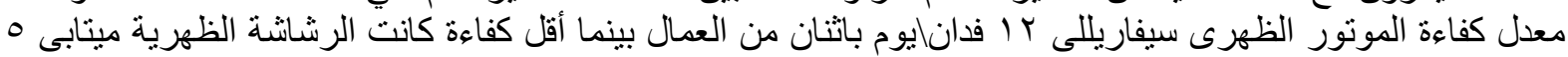

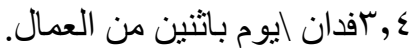

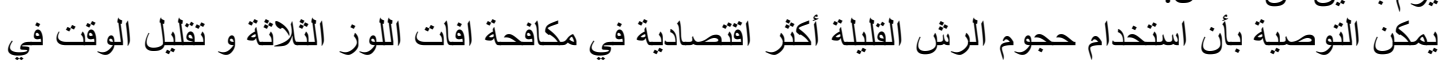
اعادة التعبئة و تحقيق تجانس محلول الرش على نباتات القطن المعاملة نوقير الفاقد من الرش على نبات التباتات القطن. 